\title{
The Effect of Spacing on the Vortex-Induced Vibrations of Two Tandem
}

Flexible Cylinders

Enhao Wang, Qing Xiao, Qiang Zhu and Atilla Incecik

NOTICE: this is the author's version of a work that was accepted for publication in Physics of Fluids. Changes resulting from the publishing process, such as final peer review, editing, corrections, structural formatting, and other quality control mechanism may not be reflected in this document. Changes may have been made to this work since it was submitted for publication. This manuscript was accepted for publishing on 09 July 2017. 


\title{
The effect of spacing on the vortex-induced vibrations of two tandem flexible cylinders
}

\author{
Enhao Wang ${ }^{1}$, Qing Xiao ${ }^{1 a)}$, Qiang Zhu² and Atilla Incecik ${ }^{1}$ \\ ${ }^{1}$ Department of Naval Architecture, Ocean and Marine Engineering, University of Strathclyde, Glasgow G4 OLZ, \\ Scotland, UK \\ ${ }^{2}$ Department of Structural Engineering, University of California San Diego, La Jolla, CA 92093-0085, USA
}

Vortex-induced vibrations (VIV) of two flexible cylinders arranged in tandem are studied using a two-way fluidstructure interaction (FSI) method with different spacing ratios $\left(S_{x} / D\right)$ at Reynolds number $\mathrm{Re}=500$ using a twoway fluid-structure interaction (FSI) method. The main objective of this study is to investigate the effect of spacing on the hydrodynamic interactions and the VIV responses of these cylinders. The responses of the two flexible cylinders are found to be similar to the classical VIV responses at small $S_{x} / D$. Once $S_{x} / D$ is large enough for the vortices to become detached from the upstream cylinder, the response of the upstream cylinder is similar to the typical VIV response whereas the downstream cylinder undergoes wake-induced vibration (WIV). The characteristics of the response of the downstream cylinder in the present study are similar to those of the first two response regimes classified by previous researchers. The third regime is not observed for the flexible downstream cylinder with both ends fixed. The two changes in the phase relation between the cross-flow displacements of the two tandem flexible cylinders are discovered to be linked with the initial-upper branch transition and the upperlower branch transition, respectively. The correlation lengths of the two flexible cylinders decrease significantly in the transition range between the upper and lower branches. Three modes of vortex shedding $(2 \mathrm{~S}, \mathrm{P}+\mathrm{S}$ and $2 \mathrm{P})$ have been identified in the present study. The upper-branch $2 \mathrm{P}$ mode is found to be associated with largeamplitude vibration of the upstream cylinder and the $\mathrm{P}+\mathrm{S}$ mode is observed to be related to large-amplitude vibration of the downstream cylinder for $S_{x} / D=3.5$ and 5 . On the other hand, the lower-branch $2 \mathrm{P}$ mode leads to small-amplitude vibration of the downstream cylinder in the post-lock-in range at $S_{x} / D=2.5$. The relative phase shifts of the sectional lift coefficients on different spanwise cross sections can be attributed to the variation of the vortex shedding flow along the flexible cylinders, and these phase shifts result in poor phasing between the forces and the displacements which is related to the decrease of the correlation lengths.

\footnotetext{
a) Author to whom correspondence should be addressed. Electronic mail: qing.xiao@strath.ac.uk. Tel: +44 01415484779.
} 


\section{INTRODUCTION}

Vortex-induced vibration (VIV) of a single cylinder has been extensively studied by various researchers over the past few decades and comprehensive reviews can be found in Blevins ${ }^{1}$, Sarpkaya $^{2}$, Bearman $^{3}$, Williamson and Govardhan $^{4}$, Gabbai and Benaroya ${ }^{5}$, Sumer and Fredsøe ${ }^{6}$, Bearman ${ }^{7}$, Wu, et al. ${ }^{8}$, Païdoussis, et al. ${ }^{9}$ and Triantafyllou, et al. ${ }^{10}$. However, there are many engineering problems which involve clusters of cylindrical structures being arranged in tandem configurations. The examples include heat exchanger tubes, chimney stacks, offshore structures and transmission lines. Due to its practical significance, more and more studies are carried out on VIV of multiple tandem cylinders.

Compared to a single cylinder, the flow past two tandem cylinders is much more complex due to the introduction of a new geometric parameter, i.e., the spacing between the cylinders $\left(S_{x}\right)$. There are a number of flow regimes depending on the spacing between the two cylinders ${ }^{11,12}$. For two stationary cylinders in tandem arrangement, three major types of flow patterns have been identified. When the two cylinders are placed in very close proximity, the two cylinders behave as a single body. The separated shear layers from the upstream cylinder wrap around the downstream cylinder without reattaching onto its surface and form a single Kármán vortex street in the wake. With the increase of the spacing, the separated shear layers from the upstream cylinder reattach onto the surface of the downstream cylinder. When the spacing between the cylinders increases beyond a critical spacing, vortex shedding occurs in the gap region between the two cylinders so that the wake behind the cylinders is a combination of the wakes of the two cylinders.

Some experimental studies have been conducted to investigate the interference between two rigid circular cylinders undergoing VIV. Hover and Triantafyllou ${ }^{13}$ and Assi, et al. ${ }^{14,15}$ considered the effect of the upstream cylinder wake on the response of the downstream cylinder. In their investigations, the upstream cylinder was stationary whilst the downstream cylinder was elastically mounted and free to vibrate in the cross-flow direction only. In Hover and Triantafyllou ${ }^{13}$, the downstream cylinder was placed at $4.75 D$ behind the upstream cylinder and the Reynolds number was $\mathrm{Re}=3 \times 10^{4}$. Large-amplitude galloping response was observed for the downstream cylinder. The results also indicated that the frequency lock-in began at a low reduced velocity $\left(V_{r}\right)$ which was nearly the same as a single cylinder and its range extended to a $V_{r}$ of at least 17 . A phase change in the lift force, which was typically associated with the frequency lock-in, occurred at higher $V_{r}$. The force spectra suggested that the shedding from the upstream cylinder was not affected by motions of the downstream cylinder. Assi, et al. ${ }^{14}$ elaborated the excitation mechanism of wake-induced vibration (WIV) of the downstream cylinder. The Reynolds number in their study was up to $3 \times 10^{4}$. They suggested that the WIV of the downstream cylinder was excited by the unsteady vortex-structure interactions between the body and the upstream wake. In a recent research by Assi, et al. ${ }^{15}$, the authors investigated how the cylinder responded to the vortex-structure interactions excitation. They introduced the concept of wake stiffness and concluded that it was the wake stiffness phenomenon that defined the character of the WIV response. Zdravkovich ${ }^{16}$ studied VIV of two elastically mounted rigid cylinders in tandem arrangement with different centre-to-centre spacing ratios $\left(S_{x} / D\right)$ in the Re range of $10^{4}-10^{5}$. It was observed that for a very small $S_{x} / D$ up to 1.1 , the two cylinders acted as a single body. With a slight increase of $S_{x} / D$ up to 1.6 , the shear layers from the upstream cylinder reattached onto the downstream cylinder. A bistable regime was found when $S_{x} / D$ varied from 2.5 to 4 , which indicated the minimum spacing required for the upstream cylinder to have regular vortex shedding. In this regime, the oscillation amplitudes of the upstream cylinder were 
larger than those of the downstream cylinder. For $S_{x} / D$ larger than 4 , the response of the upstream cylinder was smaller and less regular than that of the downstream cylinder.

The interference has also been considered for vibrating flexible cylinders. Brika and Laneville ${ }^{17,18}$ investigated VIV of a long flexible circular cylinder immersed in the wake of an identical stationary cylinder. $S_{x} / D$ between the two cylinders ranged from 7 to 25 and Re ranged from 5000 to 27000 . It was found that the response of the flexible cylinder was no longer hysteretic and showed a single branch with a wider synchronization region which decreased with the increase of the spacing between the two cylinders. The onset of the synchronization shifted to higher $V_{r}$ compared to the case of an isolated cylinder. Brika and Laneville ${ }^{17}$ also considered the case in which both cylinders were allowed to vibrate. In that case, the response of the downstream cylinder became hysteretic. Huera-Huarte and Bearman ${ }^{19}$ and Huera-Huarte and Gharib ${ }^{20}$ experimentally studied the vortex- and wakeinduced vibrations of two tandem flexible cylinders with near and far wake interferences in a Re range up to 12000. It was found that the responses of both cylinders showed classical VIV resonance when $V_{r}$ was close to the typical lock-in reduced velocities. When $S_{x} / D$ is small, the maximum vibration amplitude of the upstream cylinder is higher than that of the downstream cylinder and the maximum vibration amplitude of the upstream cylinder increased with the decrease in spacing. For a large $S_{x} / D$, the response of the downstream cylinder exhibited non-classical VIV resonance with large amplitudes at high reduced velocities.

Apart from the experiments, there have also been a number of numerical studies on VIV of two tandem cylinders. The majority of existing numerical studies were two-dimensional (2D) and focussed on one-degree-offreedom (1DOF) cross-flow motions of rigid cylinders. Carmo, et al. ${ }^{21}$ conducted 2D numerical simulations of the flow around two tandem circular cylinders at $\mathrm{Re}=150$. The upstream cylinder was fixed and the downstream cylinder was free to vibrate in the transverse direction. $S_{x} / D$ was varied from 1.5 to 8 . Compared to an isolated cylinder, the downstream cylinder was found to have higher maximum amplitudes and wider lock-in ranges. The vibration amplitudes for higher $V_{r}$ beyond the lock-in range were very significant. Carmo, et al. ${ }^{22}$ investigated VIV of a cylinder which was completely free to move in the cross-flow direction (i.e., with no spring or damper attached to it) subject to the wake of an identical stationary cylinder. A fixed spacing ratio $S_{x} / D=4$ was considered and Re varied from 100 to 645 . Three different regimes were identified in their 2D simulations. A monotonically decreasing amplitude with increasing $R e$ was observed in the first regime $(90 \leq \operatorname{Re} \leq 165)$. The second regime was characterised by significant scatter in the vibration amplitude and its range is from $\operatorname{Re} \approx 180$ to $\operatorname{Re}=360$. Gradual transition between the second and third regimes took place for $360 \leq \mathrm{Re} \leq 405$. The third regime extended

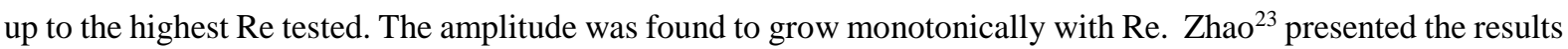
of VIV of two rigidly coupled circular cylinders in tandem arrangement with four spacing ratios ranging from 1.5 to 6 at $\operatorname{Re}=150$. The results showed that the gap between the two cylinders had a significant effect on the response. When two rigidly coupled tandem cylinders were subject to VIV, the critical spacing for vortex shedding from the upstream cylinder was significantly smaller than that for two tandem stationary cylinders. The vortex shedding from the upstream cylinder took place at $S_{x} / D=2$ in the lock-in range and the lock-in range was found to be narrower than that of a single cylinder for $S_{x} / D=1.4$ and 2, and wider for $S_{x} / D=4$ and 6 . Ding, et al. ${ }^{24}$ used $2 \mathrm{D}$ unsteady Reynolds-averaged Navier-Stokes (RANS) equations with the Spalart-Allmaras turbulence model to study the effect of tandem spacing on VIV of two cylinders with passive turbulence control in the Re range of $30000-100000$. The spacing between the cylinders varied from $2 D$ to $6 D$. The numerical simulation successfully predicted all the ranges of responses including VIV and galloping. Furthermore, the results agreed well with the 
experimental measurements. The influence of the downstream cylinder on the amplitude and frequency responses of the upstream cylinder was found to be negligible when the spacing is larger than $2 D$. A rising trend of the vibration amplitude of the downstream cylinder was observed in all the cases when $2 \times 10^{4}<\operatorname{Re}<3 \times 10^{5}$. The galloping branch merged with the VIV upper-branch for spacing larger than $3 D$. Vortex structures showed significant variation in different flow regimes.

Two-degree-of-freedom (2DOF) VIV of two tandem rigid cylinders has also been studied using 2D numerical simulations. Papaioannou, et al. ${ }^{25}$ studied the effect of spacing on VIV of two rigid cylinders in tandem arrangement. The computations were carried out for $S_{x} / D$ in the range of $2.5-5$ at $\operatorname{Re}=160$. It was observed that the range of the response region of the upstream cylinder became wider with the decrease of $S_{x} / D$. The synchronization curve shifted on the $V_{r}$ axis depending on the spacing. There was an increase in the maximum vibration amplitude of the downstream cylinder when the cylinders were brought to the spacing corresponding to the reattachment regime in the stationary system. Prasanth and Mittal $^{26}$ investigated VIV of two circular cylinders in tandem arrangement with $S_{x} / D=5.5$ at $\mathrm{Re}=100$ using a $2 \mathrm{D}$ stabilised finite element method (FEM). $V_{r}$ ranged from 2 to 15. The downstream cylinder was found to undergo large-amplitude vibrations in both the in-line and cross-flow directions. Lock-in and hysteresis were observed for both the upstream and downstream cylinders. The large-amplitude vibrations of the downstream cylinder were maintained even beyond the lock-in range. The phase difference between the cross-flow displacement and the lift force went through an $180^{\circ}$ jump in the middle of the synchronization regimes of both cylinders. The flow regime was divided into five sub-regimes based on the phase difference and the flow patterns. Bao, et al. ${ }^{27}$ studied the 2DOF VIV of two tandem cylinders with varying natural frequency ratios at $\mathrm{Re}=150$ by solving the $2 \mathrm{D}$ incompressible Navier-Stokes equations using a characteristicbased-split FEM. The spacing between the two cylinders was 5D. They found that the in-line response of the downstream cylinder was more sensitive to the natural frequency ratio than that in the transverse direction. As dual resonance was excited, the vortex shedding of the upstream cylinder in the tandem arrangement might show a $\mathrm{P}+\mathrm{S}$ pattern, which strongly suppressed the vortex shedding of the downstream cylinder.

Compared to the popularity of 2D numerical studies, three-dimensional (3D) numerical simulations of VIV of two tandem cylinders have received less attention. Carmo, et al. ${ }^{21}$ compared the 3D numerical simulation results of VIV of a rigid cylinder exposed to an upstream stationary cylinder wake at $\mathrm{Re}=300$ with their $2 \mathrm{D}$ simulation results at $\operatorname{Re}=150$. For all the configurations investigated, they observed that the $3 \mathrm{D}$ results follow the same trend as the $2 \mathrm{D}$ results. However, the flow field was strongly three dimensional for most of the configurations. Therefore, they concluded that 3D simulations were strictly necessary in order to obtain accurate values of forces or structural response. Carmo, et al. ${ }^{22}$ carried out 3D numerical simulations with the same parameters as their 2D calculations in the same study. The results showed that the variation of amplitude within the Re range tested was very small. The oscillation frequency of the downstream cylinder and the shedding frequency of the upstream cylinder were constant and had the same value throughout the entire Re range. The amplitudes observed in the 3D results are smaller than those observed in the $2 \mathrm{D}$ results for the same Re which, according to the authors, was caused by the weakening of the spanwise vortices.

There are even fewer numerical studies on VIV of two tandem flexible cylinders. Chen, et al. ${ }^{28}$ simulated VIV and WIV for two vertical risers in tandem and side-by-side arrangements at Re $=8400$ using an unsteady RANS numerical method in conjunction with a chimera domain decomposition approach with overset grids. The in-line and cross-flow responses of the risers were calculated using a tensioned beam motion equation. For two vertical 
risers in tandem arrangement, the vortex street was suppressed between the risers and there was a reattachment of the shear layers to the downstream riser when $S_{x} / D=3$. When $S_{x} / D$ increased to 4 , a vortex street started to appear in the gap between the risers with intermittent reattachment of shear layers on the downstream riser surface. In general, the computed vortex patterns and dynamic responses of the risers were in good agreement with experimental data. González, et al. ${ }^{29}$ presented the results of their numerical simulation attempts for the experimental campaigns on a tandem arrangement of flexible cylinders by Huera-Huarte, et al..$^{30}$ at $\mathrm{Re}=16000$. The numerical simulation qualitatively reproduced the physical phenomena. However, the numerical model oversimplified the mechanical complexity of the flexible cylinder and the application of the axial tensions caused the numerical instability of the computation. Therefore, the authors suggested that future research was required in order to make a quantitative comparison with the experimental data.

Overall, VIV of two elastically mounted rigid cylinders in tandem arrangement has been widely studied both experimentally and numerically. Due to the complexity of the problem, there have been fewer studies on VIV of two tandem flexible cylinders and most of the existing publications are experiments. Fully 3D fluid-structure interaction (FSI) simulations of VIV of two tandem flexible cylinders which can accurately predict the responses of the cylinders as well as provide the instantaneous 3D flow structures that are difficult to obtain in the experiments are still quite limited.

In this study, cross-flow VIV of two flexible cylinders in tandem arrangement at $\mathrm{Re}=500$ is investigated using a 3D numerical method. The choice of Re stems from the consideration that the flow around the cylinders is $3 \mathrm{D}$ and it can be modelled by directly solving the 3D Navier-Stokes equations. We focussed on the $V_{r}$ range of $V_{r}=4-10$, in which the cylinders will mainly vibrate in the first mode. The main objective of the present work is to numerically study the effect of spacing on the hydrodynamic interactions and VIV responses of the two tandem flexible cylinders. Particular attention is paid to the aspects which have not been fully addressed by previous studies such as the correlation lengths and the time-dependent 3D flow structures.

\section{NUMERICAL METHODS AND PROBLEM DESCRIPTION}

The FSI simulation in the present study is carried out using the ANSYS MFX multi-field solver ${ }^{31}$. The governing equations for the flow around the flexible cylinders are the 3D unsteady incompressible Navier-Stokes equations. The Arbitrary Lagrangian-Eulerian (ALE) scheme is employed to deal with the moving boundaries of the flexible cylinders. The ALE form of the governing equations in the Cartesian coordinate system can be expressed as

$$
\begin{gathered}
\frac{\partial u_{i}}{\partial x_{i}}=0, \\
\frac{\partial u_{i}}{\partial t}+\left(u_{j}-\hat{u}_{j}\right) \frac{\partial u_{i}}{\partial x_{j}}=-\frac{1}{\rho} \frac{\partial p}{\partial x_{i}}+v \frac{\partial^{2} u_{i}}{\partial x_{j}^{2}},
\end{gathered}
$$

where $x_{i}$ represents the Cartesian coordinate with $\left(x_{1}, x_{2}, x_{3}\right)=(x, y, z), u_{i}$ is the velocity component in the $x_{i^{-}}$ direction, $\hat{u}_{i}$ is the grid velocity component in the $x_{i}$-direction, $p$ is the pressure, $t$ is the time, $\rho$ is the fluid density and $v$ is the kinematic viscosity of the fluid. 
The Navier-Stokes equations are discretised with an element-based finite volume method (FVM) ${ }^{32}$. RhieChow interpolation ${ }^{33}$ is used to obtain pressure-velocity coupling on collocated grids. A second-order backward Euler scheme is adopted for the temporal discretisation and a high resolution scheme is used as the convection scheme which can be cast in the following form.

$$
\varphi_{i p}=\varphi_{u p}+\beta \nabla \varphi \cdot \Delta \mathbf{r}
$$

where $\varphi_{u p}$ is the value at the upwind node, $\nabla \varphi$ is the control volume gradients evaluated by the Gauss' divergence theorem and $\Delta \mathbf{r}$ is the vector from the upwind node to the integration point $i p$. In the high resolution scheme, a nonlinear recipe for $\beta$ based on the boundedness principles by Barth and Jespersen ${ }^{34}$ is used.

FIG. 1(a) shows the computational domain used in the computational fluid dynamics (CFD) simulation. The origin of the Cartesian coordinate system is located at the centre of the bottom end of the upstream cylinder as shown in FIG. 1(a). The length of the computational domain is $40 D$ in the streamwise direction ( $x$-direction) with the upstream cylinder located at $10 \mathrm{D}$ downstream the inlet boundary. The width of the domain in the cross-flow direction ( $y$-direction) is 20D and the computational domain size in the spanwise direction ( $z$-direction) is set to the cylinder length which is $12 D$. The computational mesh for $S_{x} / D=5$ used in the CFD simulation is shown in FIG. 1(b). The boundary conditions for the governing equations are as follows. The surfaces of the cylinders are assumed to be smooth, where no-slip boundary conditions are employed. Apart from the no-slip boundary conditions, the cylinder surfaces are also set to be fluid-solid interfaces where force and displacement data are transferred. The inlet velocity boundary condition is set to be the same as the freestream velocity. At the outflow boundary, the gradients of the fluid velocity in the streamwise direction are set to zero. On the two transverse boundaries, the velocity in the direction normal to the boundary is zero and a periodic boundary condition is imposed on the top and bottom boundaries. As for the pressure boundary conditions, the normal gradient of pressure is zero on the cylinder surfaces, the inlet boundary and the transverse boundaries. The pressure at the outflow boundary is given a reference value of zero.

In the present study, the two flexible cylinders are modelled as beams fixed at the two ends and they are free to vibrate in the transverse direction. The vibrations of the cylinders satisfy the Euler-Bernoulli beam theory. The transverse motions of the two flexible cylinders can be described as

$$
\frac{\partial^{2}}{\partial z^{2}}\left[E I \frac{\partial^{2} y}{\partial z^{2}}\right]+m \frac{\partial^{2} y}{\partial t^{2}}+c \frac{\partial y}{\partial t}=F_{y},
$$

where $E$ is the Young's modulus, $I$ is the moment of inertia, $m$ is the mass per unit length, $c$ is the structural damping, $z$ is the undeflected cylinder axial coordinate, $y$ is the transverse displacement and $F_{y}$ is the hydrodynamic force in the transverse direction. The boundary conditions for Eq. (4) in the case of a beam fixed at the two ends are:

$$
\begin{array}{ll}
y(0, t)=0, y(L, t)=0 & \forall t, \\
\frac{\partial y(0, t)}{\partial z}=0, \frac{\partial y(L, t)}{\partial z}=0 & \forall t .
\end{array}
$$

A finite element method is used to discretise the finite element analysis (FEA) models and the corresponding global equation system is given by

$$
M \ddot{q}+C \dot{q}+K q=F
$$


where $\mathbf{q}$ is the nodal displacement vector and a dot denotes differentiation with respect to time. The global mass, damping and stiffness matrices (M, $\mathbf{C}$ and $\mathbf{K}$ ) are the collective effects of the individual elements' mass, damping and stiffness matrices $\left(\mathbf{M}^{e}=\iiint \mathbf{N}^{T} \rho_{s} \mathbf{N} \mathrm{d} x \mathrm{~d} y \mathrm{~d} z, \mathbf{C}^{e}=\iiint \mathbf{N}^{T} c \mathbf{N} \mathrm{d} x \mathrm{~d} y \mathrm{~d} z\right.$ and $\mathbf{K}^{e}=\iiint \mathbf{B}^{T} \mathbf{E B} \mathrm{d} x \mathrm{~d} y \mathrm{~d} z$, where $\mathbf{N}$ is the matrix of shape functions, $\rho_{s}$ is the structural density, $\mathbf{B}$ is the strain-displacement matrix and $\mathbf{E}$ is the elasticity matrix) derived from the principle of virtual work. $\mathbf{F}$ is the hydrodynamic force vector. The governing equation is solved using the Hilber-Hughes-Taylor (HHT) method ${ }^{35}$ with a second-order accuracy.

A 3D 20-node solid element which exhibits quadratic displacement behaviour is used for the discretisation of the FEA models of the flexible cylinders. The element is defined by 20 nodes having three degrees of freedom per node: translations in the nodal $x$-, $y$-and $z$-directions ${ }^{36}$. FIG. 2(a) and (b) show the finite elements of the two tandem flexible cylinders for $S_{x} / D=5$ in the initial configuration and after deflections, respectively. In the solid domain, the surfaces of the cylinders are also set to be fluid-solid interfaces for data transfer.

To accommodate the motions of the flexible cylinders, the displacement diffusion model ${ }^{37-39}$ for mesh motion is adopted. The displacements of the mesh points are calculated based on the following equation:

$$
\nabla \cdot\left(\Gamma \nabla S_{y}\right)=0
$$

where $S_{y}$ represents the displacements of the nodal points in the cross-flow direction, $\Gamma$ is the mesh stiffness. In this study, in order to avoid excessive deformation of the near-wall elements, the parameter $\Gamma$ is set to be $\Gamma=1 / \forall^{2}$ where $\forall$ is the control volume size.

A two-way explicit approach is utilized for the FSI simulation in the present study. Detailed solution procedures of this approach can be described as follows. At the beginning of each time step, the flow equations are solved to obtain the forces on the flexible cylinders. Then the forces are interpolated to the FEA mesh using the conservative interpolation and the global equation system for structural dynamics is solved to obtain the quantities of cylinder motions. After that, the displacements are interpolated to the CFD mesh with the profile preserving interpolation and the positions of the mesh points are calculated and updated using Eq. (7). The next time step begins with solving the flow equations on the updated mesh. Such an FSI loop is repeated until the last time step of the simulation.

VIV of two tandem flexible cylinders is investigated numerically. Two identical flexible cylinders with diameter $D$ and length $L$ are aligned in the direction of the flow in their rest positions. To simplify the problem, the vibrations of the cylinders are confined to the cross-flow direction. Similar simplifications have been adopted by previous researchers such as Evangelinos and Karniadakis ${ }^{40}$, Evangelinos, et al. ${ }^{41}$ and Xie, et al..$^{42}$ to study VIV of a flexible cylinder. The length-to-diameter ratio is $L / D=12$, which allows the discretisation of the cylinder span with fine mesh to resolve the characteristics of the flow with acceptable computational efforts. Moreover, $L / D=12$ is also comparable to $L / D$ values used by Evangelinos, et al ${ }^{41}$ and Xie, et al. ${ }^{42}$ in their numerical studies. A moderate mass ratio $m^{*}=4 m / \rho \pi D^{2}=10$ is considered. The influence of the structural damping is mainly reflected in the maximum vibration amplitude and in order to maximize the vortex-induced responses of the flexible cylinders, the structural damping is set to zero. The Reynolds number is defined as $\operatorname{Re}=V D / v$ where $V$ is the freestream velocity and a constant Reynolds number $\mathrm{Re}=500$ is adopted in the present simulation. The reduced velocity $V_{r}=V / f_{1} D$ ranges from 4 to 10 . Here, $f_{1}$ is the fundamental natural frequency (i.e., the first

eigenfrequency) of the flexible cylinder and for a beam with two fixed ends, $f_{1}=\frac{1}{2 \pi}\left(1+\frac{\pi}{2}\right)^{2} \sqrt{\frac{E I}{m L^{4}}}$. Since $V$ is 
fixed in the present study, the bending stiffness $E I$ is varied in each simulation to obtain the corresponding $f_{1}$ of the desired $V_{r}$. In order to study the effect of spacing on the VIV of two tandem flexible cylinders, three different centre-to-centre spacing ratios are selected, i.e., $S_{x} / D=2.5,3.5$, and 5 as in Papaioannou, et al. ${ }^{25}$. These three spacing ratios are based on different regimes in the stationary system. $S_{x} / D=2.5$ belongs to the reattachment regime where the shear layers from the upstream cylinder reattach onto the surface of the downstream cylinder; $S_{x} / D=5$ represents the binary-vortex regime where the separated shear layers of the upstream cylinder roll up in the gap region and a binary vortex street is formed behind the downstream cylinder consisting of the interacting wakes of the two cylinders and the spacing $S_{x} / D=3.5$ is very close to the critical spacing of transition from the reattachment regime to the binary-vortex regime.

To confirm the flow regimes for the three spacing ratios predicted by the present methods, flow past two tandem stationary cylinders at $\mathrm{Re}=500$ was simulated and the spanwise mean drag coefficients $\left(C_{\text {Dmean }}\right)$ were compared with the numerical results of Papaioannou, et al. ${ }^{43}$ in FIG. 3. It can been seen that the present results are in good agreement with those of Papaioannou, et al. ${ }^{43}$. In the reattachment regime, $C_{D m e a n}$ of the upstream cylinder decreases with the increase of the spacing and the value of $C_{\text {Dmean }}$ experiences a jump at the critical spacing. This jump is more pronounced on $C_{\text {Dmean }}$ of the downstream cylinder, which changes from negative to positive. FIG. 4 and FIG. 5 show the 3D flow structures and the vortex shedding at $z / L=0.5$ around stationary cylinders. The present results agree with the descriptions of the different flow patterns for two tandem stationary cylinders by Igarashi ${ }^{12}$, Igarashi ${ }^{44}, \mathrm{Zdravkovich}^{11}$, Papaioannou ${ }^{45}, \mathrm{Xu}$ and Zhou ${ }^{46}$, Zhou and $\mathrm{Yiu}^{47}$, Carmo, et al. ${ }^{48}$, Carmo, et al. ${ }^{49}$ and Sumner ${ }^{50}$.

\section{VALIDATION AND MESH DEPENDENCY STUDY}

The FSI solver has been used in our previous study ${ }^{38}$ to investigate VIV of a vertical riser with $L / D=481.5$ in uniform and linearly sheared currents and the numerical results were compared with the ExxonMobil vertical riser model test results obtained at the MARINTEK by Lehn ${ }^{51}$. The numerical results were in good agreement with the experimental data. In the present study, further investigations on cross-flow VIV of a single flexible cylinder with $m^{*}=10, L / D=12$ and two fixed ends at $\operatorname{Re}=1000$ were conducted using the present numerical methods and the results are compared with the numerical results by Xie, et al. ${ }^{42}$ in FIG. 6 . It demonstrates that the present numerical methods are capable of accurately predicting VIV responses of flexible cylinders at early subcritical Reynolds numbers.

According to a series of publications on 3D numerical simulations of VIV of an elastically mounted circular cylinder $^{37,52,53}$, the important mesh parameters in the CFD simulation are the number of nodes along the circumference of the cylinder $N_{c}$, the minimum mesh size next to the cylinder surface in the radial direction $\Delta r$ and the mesh size in the spanwise direction of the cylinder $\Delta z$. Similarly, the important mesh parameter in the FEA of the present FSI simulations is the number of segments along the cylinder span $N_{z}$. In order to evaluate the dependency of the numerical results on the CFD mesh parameters, simulations were carried out for VIV of a single flexible cylinder with $m^{*}=10$ and $L / D=12$ at $V_{r}=6$ and $\operatorname{Re}=500$ with three different CFD mesh systems while using the same FEA mesh with $N_{z}=30$. The results computed using the different CFD meshes are tabulated in Table 1. As shown in the table, the maximum difference of $3.36 \%$ occurs in the value of the spanwise root mean square (rms) lift coefficient $C_{\text {Lrms }}$ between CFDM1 and CFDM2, whereas the maximum difference between 
CFDM2 and CFDM3 reduces to $1.4 \%$. It can be concluded that the combination of $N_{c}=120, \Delta r=0.001 D$ and $\Delta z$ $=0.1 D$ is sufficient for the CFD simulation of VIV of flexible cylinders in the present study. Then, CFDM2 was used to study the dependency of the numerical results on the FEA mesh parameter. Three FEA meshes are generated with $N_{z}=15,30$ and 60. The comparison of the results computed with different FEA mesh parameters is shown in Table 2. The maximum difference between FEAM1 and FEAM2 is observed in the spanwise rms drag coefficient $C_{D r m s}$ and takes the value of $1.14 \%$. In contrast, the maximum difference between FEAM2 and FEAM3 is $0.55 \%$ which appears in $C_{L r m s}$. Based on the FEA mesh dependency test results, $N_{z}=30$ is used in the present study.

\section{RESULTS AND DISCUSSIONS}

Numerical simulation was performed for VIV of two flexible cylinders in tandem arrangement with three different spacing ratios $\left(S_{x} / D=2.5,3.5\right.$ and 5) at an early subcritical Reynolds number $\operatorname{Re}=500$ for a reduced velocity range $V_{r}=4-10$. The results are compared with those of a single flexible cylinder to investigate the effect of spacing on the hydrodynamic interactions and the VIV responses of the two tandem flexible cylinders.

\section{A. Dominant modes}

In order to investigate the dominant modes of the flexible cylinders and the contributions of each vibration mode to the overall dynamic responses, the modal amplitudes are computed using the same modal decomposition method as in Huera-Huarte and Bearman ${ }^{19,54}$. The modal analysis is based on the fact that the riser response can be expressed in matrix form as a linear combination of its modes.

$$
\mathbf{y}(z, t)=\mathbf{\Phi}(z) \mathbf{y}_{M}(t),
$$

where $\boldsymbol{\Phi}=\left[\phi_{1}, \phi_{2}, \ldots, \phi_{n}\right]$ is the displacement modal-shape matrix which is built by stacking the nondimensional mode shapes which are normalized to be 1 at its maximum as columns. $\mathbf{y}(z, t)=\left[y_{1}\left(z_{1}, t\right), y_{2}\left(z_{2}, t\right), \ldots, y_{n}\left(z_{n}, t\right)\right]$ is the measured displacement matrix whose row vectors are the time series of the measured displacements along the cylinder. $\mathbf{y}_{M}(t)$ is the modal contribution matrix containing the time series of each mode's contribution to the overall response as the row vectors $\mathbf{y}_{M}(t)=\left[y_{M 1}(t), y_{M 2}(t), \ldots, y_{M n}(t)\right]$. We have

$$
\mathbf{y}_{M}(t)=\boldsymbol{\Phi}^{-1}(z) \mathbf{y}(z, t) \text {. }
$$

FIG. 7 illustrates the variation of the modal amplitudes of the first three modes with $V_{r}$. It can be observed from the figure that the dominant mode in most cases is the first mode. The contribution of the second mode is relatively small compared to that of the first mode at low $V_{r}$, but this increases gradually with the increase of $V_{r}$. At $V_{r}=10$, the modal amplitude of the second mode overtakes that of the first mode for a single flexible cylinder. The contribution of the third mode remains small throughout the $V_{r}$ range considered. FIG. 8 shows the instantaneous nondimensional deflections of the flexible cylinders at $V_{r}=6$. As shown in FIG. 8, all the vibrations at $V_{r}=6$ demonstrate easily identifiable first mode characteristics. The instantaneous nondimensional deflections of the flexible cylinders at $V_{r}=10$ are presented in FIG. 9. The vibration of a single flexible cylinder can be seen to be dominated by the second mode. Meanwhile, the vibrations of the two tandem flexible cylinders at $V_{r}=10$ 
are not as regular as those at $V_{r}=6$. The irregularity in the vibrations of the two flexible cylinders is due to the fact that contributions of the first and the second modes are comparable at $V_{r}=10$.

\section{B. Response amplitudes}

In Huera-Huarte and Bearman ${ }^{19}$, the envelopes of the displacements are calculated using the analytical signals described by Pikovsky, et al. ${ }^{55}$ based on the use of Hilbert transforms, and the mean values of the envelopes are used to represent the vibration amplitudes $\left(A_{y} / D\right)$. The same technique is adopted in the present study to determine the vibration amplitudes of the flexible cylinders. FIG. 10 shows the time histories of the nondimensional displacements $(y / D)$ on the different cross sections along the two tandem flexible cylinders when $S_{x} / D=5$ and $V_{r}$ $=6$. The solid red lines are the envelopes of the signals and the dashed red lines represent the mean values of the envelopes inside the selected time window by which the sectional vibration amplitudes $\left(A_{y}(z) / D\right)$ are denoted. $A_{y} / D$ is determined by the maximum value of $A_{y}(z) / D$ along each cylinder. FIG. 11 shows the variation of $A_{y} / D$ with $V_{r}$ for the two flexible cylinders arranged in tandem at different $S_{x} / D$. The results for a single flexible cylinder are also included for comparison. As the dominant mode in most of the cases considered in the present study is the first mode, the majority of $A_{y} / D$ is observed at $z / L=0.5$. The exceptions appear at $V_{r}=10$ when the contribution of the second mode is of a similar order of magnitude to that of the first mode. As shown in FIG. 9, for two flexible cylinders in tandem arrangement, $A_{y} / D$ appears around $z / L=0.4$ and it shifts to $z / L \approx 0.3$ in the case of a single flexible cylinder where the second mode vibration dominates. In general, the $A_{y} / D$ response curves in FIG. 11 are similar to the modal amplitude response curves of the first mode in FIG. 7 due to the fact that the first mode is the dominant mode in most cases.

The maximum vibration amplitudes $\left(A_{y m} / D\right)$ of the upstream cylinder for $S_{x} / D=3.5$ and 5 are observed at an identical reduced velocity $V_{r}=6$ with a magnitude of $A_{y m} / D \approx 0.76$ which is similar to $A_{y m} / D$ of a single flexible cylinder. As the spacing decreases to $S_{x} / D=2.5, A_{y m} / D$ of the upstream cylinder increases to 0.87 . This increase in $A_{y m} / D$ of the upstream cylinder with the decrease of $S_{x} / D$ was also observed by Huera-Huarte and Bearman ${ }^{19}$ in their experimental study with Re up to 12000 and by Papaioannou, et al. ${ }^{25}$ in their $2 \mathrm{D}$ numerical simulation at Re $=160$. Compared to the upstream cylinder, the effect of the hydrodynamic interactions between the two tandem flexible cylinders on the vibration amplitude responses of the downstream cylinder is more obvious. The maximum vibration amplitudes of the downstream cylinder for $S_{x} / D=3.5$ and 5 are found to be $\operatorname{similar}\left(A_{y m} / D \approx\right.$ 1). This is different from the results of Papaioannou, et al. ${ }^{25}$ in which the maximum vibration amplitude of the downstream cylinder in the case $S_{x} / D=3.5$ is similar to that at $S_{x} / D=2.5$. It is believed that this difference can be attributed to the difference in Re. Papaioannou, et al. ${ }^{25}$ considered a relatively low Reynolds number $\operatorname{Re}=160$. In contrast, the Reynolds number in the present simulation is $\mathrm{Re}=500$. According to Sumner ${ }^{50}$, the critical spacing $\left(S_{x} / D\right)_{c r}$ is particularly sensitive to the Reynolds number. Ljungkrona and Sundén ${ }^{56}$ reported that $\left(S_{x} / D\right)_{c r}$ at $\operatorname{Re}=$ 160 is approximately 4.54 and decreases to $\left(S_{x} / D\right)_{c r} \approx 3.7$ when Re increases to 500 . Therefore, $S_{x} / D=3.5$ is further below the critical spacing ratio in the study of Papaioannou, et $a l .{ }^{25}$ than in the present study resulting in $A_{y m} / D$ at $S_{x} / D=3.5$ in their study being similar to that at $S_{x} / D=2.5$.

In the present study, when $S_{x} / D$ falls to $2.5, A_{y m} / D$ of the downstream cylinder increases to 1.1 and it shifts to a higher reduced velocity $V_{r}=7$ due to the shielding effect of the upstream cylinder. When $V_{r}=8-10$, for the three spacing ratios considered, the upstream cylinder shows a typical VIV response with very small vibration 
amplitudes at high $V_{r}$. In terms of the downstream cylinder, a lower-branch VIV response is observed for $S_{x} / D=$ 2.5 and 3.5 due to the fact that the vortices impinging on the downstream cylinder are connected to the shear layers of the upstream cylinder as illustrated by the spanwise vorticity $\left(\omega_{z}=\partial u_{2} / \partial x_{1}-\partial u_{1} / \partial x_{2}\right)$ contours in FIG. 23(a) and (b). In contrast, at $S_{x} / D=5$, the completely detached vortices from the upstream cylinder in FIG. 23(c) cause the wake-induced vibration (WIV) of the downstream cylinder with large-amplitude vibration being maintained up until the maximum $V_{r}$ considered in the present study. Assi, et al. ${ }^{15}$ classified the response of an elastically mounted downstream cylinder into three regimes: (i) before the vortex shedding frequency $f_{v}=$ the natural frequency of the cylinder $f_{n}$, when the Strouhal number St $=0.2$ is approaching $f_{n}$, the $A_{y} / D$ response resembles the typical VIV response; (ii) the second regime, between $f_{v}=f_{n}$ and the equivalent natural frequency of the wake stiffness $f_{w}=f_{n}$, is marked by a steep slope in the $A_{y} / D$ response curve and (iii) the third regime, beyond $f_{w}=f_{n}$ is characterised by a change of the slope in the response curve of $A_{y} / D$. According to their descriptions, the $A_{y} / D$ response of the flexible downstream cylinder at $S_{x} / D=5$ in FIG. 11 is similar to the first two regimes and the third regime is not observed in the present study and the possible reasons will be provided in Section IV.C. We also notice that the present $A_{y} / D$ in the second regime shows a plateau instead of increasing monotonically with $V_{r}$ as shown in the experimental results of Hover and Triantafyllou ${ }^{13}$ and Assi, et al. ${ }^{14,15}$. This discrepancy can be attributed to the different treatments of Re between the present study and the experiments. In the present study, $V$ and Re are fixed and $E I$ is varied to obtain the desired $V_{r}$. In contrast, $V$, as well as Re, is usually increased in the experiment in order to increase $V_{r}$. Assi, et al. ${ }^{15}$ concluded that the increasing trend of the vibration amplitude in WIV is a direct effect of Re rather than $V_{r}$. Therefore, it is not surprising that the aforementioned difference in the $A_{y} / D$ responses in the second regime exists between the present results and the experimental data.

\section{Response frequencies}

Spectral analysis is conducted for the time histories of the displacements after the eliminations of the transient responses. The oscillation frequencies $\left(f_{\text {oy }}\right)$ are determined by the frequencies associated with the prominent peaks of the $y / D$ amplitude spectra and then normalized using $f_{1}$. FIG. 12 shows the comparison of the nondimensional oscillation frequencies $\left(f_{o y} / f_{1}\right)$ between the two flexible cylinders in tandem arrangement and a single flexible cylinder. Based on the synchronization between $f_{o y}$ and $f_{1}$, the lock-in range of the single flexible cylinder in terms of $V_{r}$ is from 4 to 7 . Within the lock-in range, $f_{o y}$ locks into $f_{1}$ of the flexible cylinder. As $V_{r}$ increases beyond the lock-in range, $f_{o y}$ follows the Strouhal line. It can be observed that the frequency response of a single flexible cylinder is similar to that of an elastically mounted circular cylinder reported by Zhao, et al. ${ }^{37}$ and Govardhan and Williamson $^{57}$. For two tandem flexible cylinders, the lock-in ranges for $S_{x} / D=2.5$ and 3.5 are identical (i.e., $V_{r}=$ $4-8$ ). When $S_{X} / D$ increases to 5 , the lock-in range of the upstream cylinder changes to $V_{r}=5-8$. The synchronization of the downstream cylinder also starts at $V_{r}=5$. However, it extends to the maximum $V_{r}$ considered in the present simulation. The $f_{\text {oy }} / f_{1}$ response of the flexible downstream cylinder at $S_{x} / D=5$ in FIG. 12 is also similar to the first two regimes described by Assi, et al. ${ }^{15}$ : in the first regime, $f_{\text {oy }}$ follows the Strouhal line; and in the second regime, $f_{\text {oy }}$ remains rather close to $f_{1}$. As mentioned in the previous section, the third regime characterised by $f_{o y}=f_{w}$ is not observed for the flexible downstream cylinder with two fixed ends in the present simulation. The reasons are as follows: unlike an elastically mounted rigid cylinder, a flexible cylinder has an 
infinite number of natural frequencies. In order for the third regime to occur, $f_{w}$ needs to dominate all of the natural frequencies of a flexible cylinder, which is unrealistic. In addition, for a flexible downstream cylinder with two fixed ends, the natural frequencies of two neighbouring vibration modes are so close that when $f_{w}$ surpasses the natural frequency of one mode, it is already within the bandwidth of the next mode. Therefore, it is expected that, with the increase of $V_{r}$, the response of the present flexible downstream cylinder will repeat the first two regimes for the different vibration modes.

Unlike the single flexible cylinder, $f_{o y}$ of the two tandem flexible cylinders in the post-lock-in range deviates from the Strouhal line, which reveals the effect of hydrodynamic interactions on $f_{\text {oy }}$ of the two tandem flexible cylinders. It should also be noted that $f_{\text {oy }}$ of the downstream cylinder in the post-lock-in range is the same as the corresponding $f_{\text {oy }}$ of the upstream cylinder for $S_{x} / D=2.5$ and 3.5. This is related to the aforementioned fact that the vortices rolling up in the gap region between the two cylinders are still connected to the upstream cylinder through the shear layers when they impinge on the downstream cylinder. Consequently, for configurations with $S_{x} / D=2.5$ and 3.5 , the oscillation frequencies of the two cylinders in the post-lock-in range are identical.

\section{Phase Differences between Displacements of Two Tandem Flexible Cylinders}

In this section the phase differences $(\phi)$ between the cross-flow displacements at $z / L=0.5$ of the two tandem flexible cylinders are analysed. The instantaneous phase difference $\phi(t)$ can be determined by using the concept of the analytical signal ${ }^{55}$, based on Hilbert transforms. In the present study, $\phi(t)$ is calculated for the cases with two cylinders vibrating at an identical frequency in the time range of $t=100-115 \mathrm{~s}$ when the responses of the two cylinders are in their steady states. In addition, $\phi(t)$ at $V_{r}=4$ is excluded as the motions of the cylinders are so small that the computed $\phi(t)$ are not reliable. In all the cases considered, $\phi(t)$ fluctuates around a constant value within the selected time range. Therefore, $\phi$ is represented by the mean value of $\phi(t)$. FIG. 13 illustrates the variations of $\phi$ with $V_{r}$ for the three spacing ratios considered in the present study. In FIG. 13, positive $\phi$ means that $y / D$ of the upstream cylinder leads that of the downstream cylinder and negative $\phi$ indicates that $y / D$ of the upstream cylinder lags that of the downstream cylinder. Based on the changes in the sign of $\phi$, it can be concluded that, for each of the three spacing ratios, the phase relation between the displacements of the two tandem flexible cylinders changes twice over the $V_{r}$ range considered. At $V_{r}=5, \phi$ is negative (i.e., $y / D$ of the upstream cylinder lags that of the downstream cylinder) for $S_{x} / D=2.5$ and 5 and it becomes positive (i.e., $y / D$ of the upstream cylinder leads that of the downstream cylinder) when $V_{r}$ increases to 6 . With the increase of $V_{r}$, the sign of $\phi$ experiences a second change, taking place in the range of $V_{r}=7-8$. As for $S_{x} / D=3.5$, the $V_{r}$ ranges associated with the two changes of the phase relation are the same as those of the other two spacing ratios, whereas the variations in phase relation are opposite. According to the discussion above, the two phase relation changes occur at reduced velocities corresponding to the left of the upper branch and the transition region between the upper and lower branches, respectively. Therefore, it can be speculated that the transitions from one mode of response to another (i.e., from the initial branch to the upper branch and from the upper branch to the lower branch) might have some bearing on the variations of the phase relation between the cross-flow displacements of the two flexible cylinders. Nevertheless, qualitative and quantitative differences in the variations of $\phi$ with $V_{r}$ are observed for different spacing ratios reflecting that the phase difference between the cross-flow displacements of the two 
tandem flexible cylinders depends on $V_{r}$ and $S_{x} / D$. This conclusion agrees with that of Laneville and Brika ${ }^{58}$ drawn from their wind tunnel test on VIV of two tandem flexible cylinders.

\section{E. Hydrodynamic forces}

The total drag and lift coefficients are defined as $C_{D}=F_{x} /\left(0.5 \rho V^{2} D L\right)$ and $C_{L}=F_{y} /\left(0.5 \rho V^{2} D L\right)$, respectively. Here, $F_{x}$ and $F_{y}$ represent the total in-line and cross-flow hydrodynamic forces computed by summing the dot product of the pressure and viscous forces with the specified force vector over the cylinder surface. FIG. 14(a) and (b) show the variation of the mean drag coefficients $\left(C_{D m e a n}\right)$ with $V_{r}$. The constant horizontal lines indicate the values of each cylinder in the stationary system for each $S_{x} / D$. At $V_{r}=4$, the vibration amplitudes of both cylinders for the three spacing ratios considered are very small. The flow around the two tandem flexible cylinders in FIG. 20 at each $S_{x} / D$ are essentially similar to that around two tandem stationary cylinders in FIG. 5. Therefore, the mean drag coefficients of both cylinders are close to the values corresponding to the stationary system. Similar to the single flexible cylinder case, the mean drag coefficients of both cylinders reach their maximum values at reduced velocities where the maximum vibration amplitudes appear. For $V_{r}$ in the post-lock-in range, the values of $C_{\text {Dmean }}$ of the upstream cylinder at the three spacing ratios nearly coincide with each other. Overlaps in the $C_{\text {Dmean }}$ response curves of the downstream cylinder are observed for $S_{x} / D=3.5$ and 5 when $V_{r} \geq 5$.

FIG. $14(\mathrm{c})$ and (d) present the variation of the rms values of the oscillating lift coefficients $\left(C_{L r m s}\right)$ with $V_{r}$. It shows that the $C_{L r m s}$ response curve of the upstream cylinder at $S_{x} / D=5$ resembles that of a single flexible cylinder indicating the negligible effect of the downstream cylinder on the upstream cylinder when $S_{x} / D=5$. The maximum $C_{\text {Lrms }}$ values of the upstream cylinder for the three spacing ratios considered appear at an identical reduced velocity $V_{r}=5$. The $C_{L r m s}$ of the upstream cylinder attains a common value when $V_{r}$ is large enough that the response of the upstream cylinder is very small for $S_{x} / D=3.5$ and 5 . This is observed because the oscillations corresponding to those reduced velocities are still large enough to allow vortices to form in the gap region. Similar $C_{L r m s}$ values of the two cylinders are observed at $V_{r}=4$ for $S_{x} / D=2.5$ and 3.5. The flow patterns in these two cases are essentially similar, namely the shear layers from the upstream cylinder reattach onto the surface of the downstream cylinder forming a single Kármán vortex street behind the downstream cylinder (FIG. 20(a) and (b)). However, large discrepancies take place in the $C_{L r m s}$ response of the upstream cylinder between $S_{x} / D=2.5$ and the other two spacing ratios for $V_{r}=6-9$, which reflects that for $S_{x} / D=2.5$ the oscillations at those reduced velocities are not large enough for the vortex shedding flow in the gap region to get fully developed. As shown in FIG. 14(d), the $C_{\text {Lrms }}$ responses of the downstream cylinder for all the three spacing ratios are quite different from each other and from that of the single flexible cylinder, which highlights that the wake behind the downstream cylinder is a combination of the interacting wakes of the two cylinders.

\section{F. Correlation lengths}

The three-dimensionality of the flow in the near wake of the cylinder which determines the fluctuations of the forces acting on the cylinder is measured by the spanwise correlation length. Previous experimental and numerical studies on VIV of an elastically mounted circular cylinder indicated that there was a sharp drop in the spanwise correlation at the end of the upper branch near the transition between the upper and the lower branches, which 
does not diminish the response of the cylinder ${ }^{37,39,59-62}$. In the present study the autocorrelation function as defined in Lucor, et al. ${ }^{62}$ is used to quantify the correlation. The autocorrelation function is described as follows.

$$
R(l, t)=\frac{1}{N_{t}} \sum_{j=1}^{N_{t}}\left[\frac{\left(1 / N_{z}\right) \sum_{i=1}^{N_{z}} C_{L}\left(z_{i}, t_{j}\right) C_{L}\left(z_{i}-l_{k}, t_{j}\right)}{\left(1-N_{z}\right) \sum_{i=1}^{N_{z}} C_{L}^{2}\left(z_{i}, t_{j}\right)}\right],
$$

where $C_{L}\left(z_{i}, t_{j}\right)$ is taken to be the fluctuation of the original signal $C_{L}{ }^{*}\left(z_{i}, t_{j}\right)$ from which its mean quantity is subtracted. The signal $C_{L}\left(z_{i}, t_{j}\right)$ is given by

$$
C_{L}\left(z_{i}, t_{j}\right)=C_{L}^{*}\left(z_{i}, t_{j}\right)-\frac{1}{N_{t}} \sum_{n=1}^{N_{t}} C_{L}\left(z_{i}, t_{n}\right) .
$$

The shift $l_{k}$ in Eq. (10) is prescribed to be

$$
l_{k}=k \times l=k \times d z \text { with } d z=l / N_{z} \text { and } k=\left[0,1,2, \ldots \frac{N_{z}}{2}\right] .
$$

The correlation length $L_{C}$ is then computed by

$$
L_{C}(t)=2 \int_{0}^{\frac{L / D}{2}} R(l, t) d l .
$$

FIG. 15 shows the variation of the nondimensional correlation lengths $\left(L_{C} / D\right)$ with $V_{r}$ for flexible cylinders. In general, $L_{C} / D$ is quite large for low $V_{r}$ around 4 or 5 , representing the initial branch or the left of the upper branch. The large $L_{C} / D$ is indicative of the strong two-dimensionality of the flow at low $V_{r}$. As $V_{r}$ increases, there is a drop in $L_{C} / D$ and it reaches its minimum value between $V_{r}=6$ and 7, close to the transition region between the upper and lower branches. Finally, $L_{C} / D$ increases for higher $V_{r}$ which corresponds to the lower branch of response. Lucor, et al. ${ }^{62}$ conducted a 3D numerical study on 1DOF VIV of an elastically mounted circular cylinder and observed similar variation trends of $L_{C} / D$ with $V_{r}$. For two tandem stationary cylinders, Wu, et al. ${ }^{63}$ observed that when the two cylinders were close together $\left(S_{x} / D<3\right)$, the downstream cylinder suppressed the shedding of the upstream vortices resulting in the large correlation lengths of the upstream cylinder. As the turbulence intensity and three-dimensionality of the impinging flow on the downstream cylinder were weak, high spanwise correlation was also observed for the downstream cylinder. For most cases with two flexible cylinders in tandem arrangement subject to VIV, the correlation lengths of the two flexible cylinders at $S_{x} / D=2.5$ are larger than those for $S_{x} / D=$ 3.5 and 5, which agrees with the conclusion of $\mathrm{Wu}$, et al. ${ }^{63}$ drawn from two tandem stationary cylinders. With the increase of $S_{x} / D$, the suppression effect of the downstream cylinder on the upstream vortex shedding diminishes gradually, which leads to similar variations of the upstream cylinder correlation lengths for $S_{x} / D=3.5$ and 5 when $V_{r} \geq 5$. However, as the level and form of the velocity perturbation introduced by the presence of the upstream cylinder depend on the distance between the two cylinders ${ }^{63}$, discrepancies in the correlation lengths of the downstream cylinder are observed for $S_{X} / D=3.5$ and 5 .

The variations of the lift coefficients and the displacements along the span at $V_{r}=5,6$ and 8 are examined by plotting the contours of the sectional lift coefficients $\left(C_{L}(z)=F_{y}(z) /\left(0.5 \rho V^{2} D\right)\right.$, in which the sectional force in the cross-flow direction $F_{y}(z)$ is obtained by summing up the dot product of the pressure and viscous forces with the specified force vector over a circular cross section) and the sectional displacements $(y(z) / D)$ on the $z-t$ plane. The choice of the three reduced velocities stems from the following considerations. $V_{r}=5$ represents the large correlation lengths on the left of the upper branch. $V_{r}=6$ is near the region where the correlation lengths approach 
their minimum values and $V_{r}=8$ stands for the large correlation lengths in the lower branch. The results for a single flexible cylinder and two tandem flexible cylinders when $S_{x} / D=3.5$ are given in FIG. 16. It can be seen that, for the single flexible cylinder and the two tandem flexible cylinders, when $L_{C} / D$ is large, the distributions of $C_{L}(z)$ and $y(z) / D$ are well organised. The phase differences between $C_{L}(z)$ and $y(z) / D$ along the cylinder in each high correlation case are very regular. In contrast, when the correlation is low, $C_{L}(z)$ signals at different spanwise locations suffer from relative phase shifts despite the fact that $y(z) / D$ signals along the cylinder are nearly in phase. This is caused by the variation of the phase differences between $C_{L}(z)$ and $y(z) / D$ along the cylinder. The poor phasing between $C_{L}(z)$ and $y(z) / D$ leads to the decrease of the spanwise correlation ${ }^{62}$.

\section{G. Vortex shedding}

One of the objectives of the present study is to investigate the vortex shedding of two tandem flexible cylinders undergoing VIV. The $\lambda_{2}$ method by Jeong and Hussain ${ }^{64}$ is used to describe the $3 \mathrm{D}$ vortex structures in which $\lambda_{2}$ is the second eigenvalue of the symmetric tensor $\mathbf{S}^{2}+\mathbf{\Omega}^{2}$. Here $\mathbf{S}$ and $\boldsymbol{\Omega}$ are the symmetric and antisymmetric parts of the velocity gradient tensor $\nabla \mathbf{u}$. FIG. 17- FIG. 19 present the iso-surfaces of $\lambda_{2}=-0.1$ at $V_{r}=4,6$ and 10 . The iso-surfaces are coloured by $\omega_{z}$. It can be observed that the wake flow is entirely $3 \mathrm{D}$. The variation of the flow in the spanwise direction is stronger in the cases with smaller correlation lengths. Our previous discussions reveal that the hydrodynamic coefficients of the two tandem flexible cylinders at $V_{r}=4$ are close to those of two tandem stationary cylinders. Therefore, it is expected that the 3D vortex structures of the two tandem flexible cylinders at this $V_{r}$ should be similar to those of two tandem stationary cylinders. It can be seen from FIG. 17 that the $3 \mathrm{D}$ vortex structures of the flexible cylinders at $V_{r}=4$ does share similar features to that of the corresponding stationary system for each $S_{x} / D$ in FIG. 4. As shown in FIG. 17, when $S_{x} / D=2.5$ and 3.5, the shear layers from the upstream cylinder reattach onto the surface of the downstream cylinder. When $S_{x} / D$ increases to 5 , vortex shedding occurs in the gap region between the two cylinders and the wake behind the downstream cylinder is a combination of the wakes of the two cylinders. FIG. 18 presents the 3D vortex structures of the flexible cylinders at $V_{r}=6$. Compared to the results in FIG. 17, the variation of the flow in the spanwise direction becomes stronger in most of the cases apart from the upstream cylinder at $S_{x} / D=2.5$. In the cases of $S_{x} / D=2.5$ and 3.5 as shown in FIG. 18(a) and (b), the oscillations of the two tandem flexible cylinders provide extra time and space for the separated shear layers from the upstream cylinder to roll up into vortices. When $V_{r}$ increases to 10, the correlation of the spanwise vortices of the flexible cylinders in most of the cases becomes higher than that at $V_{r}=6$ except for the upstream cylinder at $S_{x} / D=2.5$. It is worth noting that although the vibration amplitudes of the two tandem flexible cylinders for $S_{x} / D=2.5$ and 3.5 are quite small at $V_{r}=10$, the shear layers from the upstream cylinder still roll up.

The contours of $\omega_{z}$ on three different cross sections $(z / L=0.25,0.5$ and 0.75$)$ are plotted in order to examine the variation of the flow in the spanwise direction of the flexible cylinders. The three cross sections are chosen in favour of those closer to the cylinder ends to avoid the potential end effect of the periodic boundary condition employed on the two spanwise boundaries. FIG. 20 shows $\omega_{z}$ contours of the flexible cylinders on the three cross sections when $V_{r}=4$. The vortex shedding patterns are qualitatively similar to those in FIG. 5 for stationary cylinders. When $S_{x} / D=2.5$ and 3.5, the shear layers from the upstream cylinder reattach onto the surface of the downstream cylinder. At $S_{x} / D=5$, vortex shedding occurs in the gap region between the two cylinders and a 
binary vortex street forms behind the downstream cylinder. The vortex shedding demonstrates a clear $2 \mathrm{~S}$ pattern with two single vortices being formed in one cycle as described by Williamson and Roshko ${ }^{65}$. As $V_{r}$ increases to 6 (FIG. 21), the wake flow behind the flexible cylinders becomes wider. Vortices are formed behind the upstream cylinder in the gap region and these upstream vortices interact with the shear layers of the downstream cylinder when they impinge on the surface of the downstream cylinder. Variation of the vortex shedding modes along the cylinders is observed. The vortex shedding exhibits a $2 \mathrm{~S}$ mode at $z / L=0.25$ and 0.75 where the vibration amplitudes are small. With the increase of the vibration amplitudes towards the middle sections of the cylinders $(z / L=0.5)$, a $2 \mathrm{P}$ vortex shedding mode with two pairs of vortices being formed per cycle is observed behind the upstream cylinder. Brika and Laneville ${ }^{66,67}$ were the first to show evidence of the $2 \mathrm{P}$ vortex shedding mode in free vibration. The $2 \mathrm{P}$ mode at $V_{r}=6$ in the present study is qualitatively similar to the $2 \mathrm{P}$ mode in the upper branch found by Govardhan and Williamson ${ }^{57}$ and Jauvtis and Williamson ${ }^{68}$, in which one vortex of each vortex pair is weaker than the other. This upper-branch 2P mode is associated with the large-amplitude vibration of the upstream cylinder. For the downstream cylinder, a $2 S$ mode is observed at $S_{x} / D=2.5$, whereas the vortex shedding for $S_{x} / D=3.5$ and 5 shows a $\mathrm{P}+\mathrm{S}$ mode where the cylinder sheds a single vortex and a vortex pair per cycle. FIG. 22 shows the vortex shedding on the middle sections of the two tandem flexible cylinders at different time instants in one vibration cycle when $V_{r}=6$ for $S_{x} / D=3.5$ and 5. It can be seen that a single vortex and a vortex pair are shed from the downstream cylinder when it moves to its positive and negative peaks, respectively, which confirms the $\mathrm{P}+\mathrm{S}$ vortex shedding. Although the $\mathrm{P}+\mathrm{S}$ mode is usually observed in forced vibration experiments ${ }^{69,70}$, it has also been identified in free vibration studies at reduced velocities around the maximum vibration amplitude for an elastically mounted cylinder at low $\mathrm{Re}^{27,39,71}$ as well as a flexible cylinder undergoing low mode number vibrations $^{72}$. Therefore, the large-amplitude vibration of the downstream cylinder when $S_{x} / D=3.5$ and 5 is related to the $\mathrm{P}+\mathrm{S}$ vortex shedding mode. The variation of the vortex shedding patterns along the cylinders causes the relative phase shifts $C_{L}(z)$ which subsequently leads to the decrease of the spanwise correlation. The $\omega_{z}$ contours on three different cross sections along the flexible cylinders at $V_{r}=10$ are displayed in FIG. 23. The shear layers from the upstream cylinder are observed to roll up for $S_{x} / D=2.5$ and 3.5 despite the vibration amplitudes of the two tandem flexible cylinders are quite small at $V_{r}=10$. This confirms the observation from the 3D vortex structures as shown in FIG. 19. When $S_{x} / D=2.5$, the vortex shedding patterns of the downstream cylinder vary from a $2 \mathrm{~S}$ mode at $z / L=0.25$ and 0.75 to a lower-branch $2 \mathrm{P}$ mode at $z / L=0.5$. Unlike the upper-branch $2 \mathrm{P}$ mode observed at $V_{r}=6$, the two vortices in each vortex pair of the lower-branch 2P mode at $V_{r}=10$ have almost equal strength, thus the small-amplitude vibration of the downstream cylinder at $S_{x} / D=2.5$ is related to the lower-branch 2P mode. A $2 \mathrm{~S}$ vortex shedding mode is observed for the rest of the cases in FIG. 23.

\section{CONCLUSIONS}

The effect of spacing on the cross-flow vortex-induced vibrations of two tandem flexible cylinders is numerically studied at $\mathrm{Re}=500$ using a two-way fluid-structure interaction method. A moderate mass ratio $m^{*}=$ 10 , a length-to-diameter ratio $L / D=12$ and zero structural damping are considered in the simulation. The reduced velocity ranges from 4 to 10 in which the flexible cylinders mainly vibrate in the first mode. Three spacing ratios $\left(S_{x} / D=2.5,3.5\right.$ and 5$)$ are examined. Based on the numerical results, the effect of spacing on the hydrodynamic interactions and the VIV responses of the two tandem flexible cylinders is investigated. In particular, the aspects 
which are rarely reported in previous studies such as the correlation lengths and the three-dimensional flow structures of two tandem flexible cylinders are provided. The conclusions are summarised as follows.

The upstream cylinder shows a classical VIV response for the three spacing ratios considered. As the vortices impinging on the downstream cylinder are still connected to the shear layers of the upstream cylinder in the cases of small spacing ratios, the response of the downstream cylinder is also similar to a typical VIV response. When the spacing between the two tandem flexible cylinders is large enough for the vortices to be shed from the upstream cylinder, the downstream cylinder undergoes WIV with large amplitudes at high reduced velocities and an extended lock-in range. However, in contrast to an elastically mounted downstream cylinder whose response can be divided into three regimes, only the first two regimes appear in the present study. The third response regime is not observed due to the fact that the flexible downstream cylinder with two fixed ends has an infinite number of natural frequencies and the natural frequencies of two neighbouring vibration modes are very close. As a constant Re is used in the present study, a flattened amplitude response is observed in the second response regime of the downstream cylinder.

It is found that there exist two changes of the phase relation between the cross-flow displacements of the two flexible cylinders arranged in tandem. The reduced velocity ranges associated with the phase relation changes correspond to the left of the upper branch and the transition between the upper and lower branches, respectively. Thus, the variations of phase relation between the cross-flow displacements of the two tandem flexible cylinders are presumed to be related to the transitions from the initial branch to the upper branch and from the upper branch to the lower branch. The phase differences between the cross-flow displacements of the two tandem flexible cylinders are also found to differ for different reduced velocities and spacing ratios.

At very low reduced velocities when the vibration amplitudes of the two tandem flexible cylinders are small, the flow around the two tandem flexible cylinders is essentially similar to that around two tandem stationary cylinders. Consequently, the hydrodynamic force coefficients are close to the values corresponding to the stationary system. The effect of the downstream cylinder on the upstream cylinder is nearly negligible for large spacing ratios and for small spacing ratios, the oscillations in the classical VIV resonance range are not large enough for the vortex shedding to fully develop in the gap region. For two vibrating flexible cylinders in tandem arrangement, the wake behind the downstream cylinder consists of the interacting wakes of the two cylinders.

The variation of the correlation lengths of the two tandem flexible cylinders with the reduced velocity roughly follows that of an elastically mounted circular cylinder. In particular, the correlation lengths experience a sharp drop at reduced velocities around the mode transition between the upper and lower branches. For small spacing ratios, the presence of the downstream cylinder suppresses the three-dimensionality of the upstream flow resulting in the large correlation lengths of the upstream cylinder, and the subsequent weak turbulence intensity and threedimensionality of the upstream flow consequently lead to high spanwise correlation of the downstream cylinder. Large correlation lengths are found to be associated with regular phase differences between the sectional lift forces and sectional displacements along the cylinder, whereas the decrease of the spanwise correlation is attributed to the poor phasing between the forces and the displacements.

The flow around two flexible cylinders in tandem arrangement which are subject to VIV is totally three dimensional. The flow in the spanwise direction suffers stronger variations when the correlation is poor. In the case with spacing ratios corresponding to the reattachment regime of two tandem stationary cylinders, the largeamplitude vibrations of the two tandem flexible cylinders in the resonance range provide the time and space for 
the vortices to roll up in the gap. The wake in the lock-in range are found to be wider than that in the non-lock-in range. $2 \mathrm{~S}, 2 \mathrm{P}$ and $\mathrm{P}+\mathrm{S}$ vortex shedding modes are identified in the present study. The upper-branch $2 \mathrm{P}$ mode with one vortex being stronger than the other in each vortex pair is found to be associated with the large-amplitude vibration of the upstream cylinder in the lock-in range and the $\mathrm{P}+\mathrm{S}$ mode is related to the large-amplitude vibration of the downstream cylinder for $S_{x} / D=3.5$ and 5. In contrast, the lower-branch $2 \mathrm{P}$ mode with two equalstrength vortices in each vortex pair leads to the lower-branch response of the downstream cylinder at high reduced velocity at $S_{x} / D=2.5$. Variation of the vortex shedding modes in the spanwise direction is observed in the cases with low spanwise correlation.

Overall, the present results confirmed various important conclusions obtained from previous experimental studies. Additionally, they also highlighted some new aspects such as the disappearance of the third response regime for a flexible downstream cylinder with two fixed ends at large spacing ratios, and also the transitions between different response branches (i.e., between the initial branch and the upper branch and between the upper branch and the lower branch) may explain the changes in the phase relation between the cross-flow displacements of the two flexible cylinders in tandem arrangement. Furthermore, the correlation lengths and the 3D flow structures which are difficult to obtain in the experimental studies are provided. As the present study is one of the first few numerical investigations on VIV of two tandem flexible cylinders, there are also some limitations. The Reynolds number used in the numerical simulation is fixed with the consequence that the monotonically increasing amplitude in the second response regime of the flexible downstream cylinder at large spacing ratios was not observed. A further limitation in this regard is the present study is focused on a low Reynolds number regime. The maximum vibration amplitudes will be larger if a higher Reynolds number range is considered. The Reynolds number also influences the critical spacing of the two tandem flexible cylinders. The responses of the two tandem flexible cylinders, especially when $S_{x} / D=3.5$ might change if the Reynolds number is different. Moreover, the vibrations of the two tandem flexible cylinders in the present study are confined in the cross-flow direction. As we considered a moderate mass ratio, the effect of the in-line degree of freedom on the maximum vibration amplitudes may not be very pronounced. However, the in-line motion may have an impact on other aspects of the VIV responses of the two tandem flexible cylinders as it will change the gap between the two cylinders when they vibrate. Given the limitations of the present study, future research on coupled in-line and cross-flow VIV of two tandem flexible cylinders in a higher Re range is worthwhile.

\section{ACKNOWLEDGEMENTS}

Results were obtained using the EPSRC funded ARCHIE-WeSt High Performance Computer (www.archiewest.ac.uk). EPSRC grant no. EP/K000586/1.

1. R. D. Blevins, Flow-Induced Vibration (Van Nostrand Reinhold Company, New York, USA, 1977).

2. T. Sarpkaya, "Vortex-induced oscillations," Journal of Applied Mechanics 46, 241 - 258 (1979).

3. P. W. Bearman, "Vortex shedding from oscillating bluff body," Annual Review of Fluid Mechanics 16, 195 222 (1984).

4. C. H. K. Williamson, and R. Govardhan, "Vortex-induced vibrations," Annual Review of Fluid Mechanics 36, 413 - 455 (2004). 
5. R. D. Gabbai, and H. Benaroya, "An overview of modelling and experiments of vortex-induced vibrations of circular cylinders," Journal of Sound and Vibration 282, 575 - 646 (2005).

6. B. M. Sumer, and J. Fredsøe, Hydrodynamics around Cylindrical Structures (World Scientific Publishing Co. Pte. Ltd., London, UK, 2006).

7. P. W. Bearman, "Circular cylinder wakes and vortex-induced vibrations," Journal of Fluids and Structures 27, $648-658(2011)$.

8. X. Wu, F. Ge, and Y. Hong, "A review of recent studies on vortex-induced vibrations of long slender cylinders," Journal of Fluids and Structures 28, 292 - 308 (2012).

9. M. P. Païdoussis, S. J. Price, and E. de Langre, Fluid-Structure Interactions: Cross-Flow-Induced Instabilities (Cambridge University Press, New York, USA, 2014).

10. M. S. Triantafyllou, R. Bourguet, J. M. Dahl, and Y. Modarres-Sadeghi, Vortex-Induced Vibrations (Springer International Publishing, London, UK, 2016).

11. M. M. Zdravkovich, "The effects of interference between circular cylinders in cross flow," Journal of Fluids and Structures 1, 239 - 261 (1987).

12. T. Igarashi, "Characteristics of the flow aroudn two circular cylinders arranged in tandem (1st report)," Bulletin of the JSME 24, 323 - 331 (1981).

13. F. S. Hover, and M. S. Triantafyllou, "Galloping response of a cylinder with upstream wake interference," Journal of Fluids and structures 15, 503 - 512 (2001).

14. G. R. S. Assi, P. W. Bearman, and J. R. Meneghini, "On the wake-induced vibration of tandem circular cylinders: the vortex interaction excitation mechanism," Journal of Fluid Mechanics 661, 365 - 401 (2010).

15. G. R. S. Assi, P. W. Bearman, B. S. Carmo, J. R. Meneghini, S. J. Sherwin, and R. H. J. Willden, "The role of wake stiffness on the wake-induced vibration of the downstream cylinder of a tandem pair," Journal of Fluid Mechanics 718, 201 - 245 (2013).

16. M. M. Zdravkovich, "Flow induced oscillations of two interfering circular cylinders," Journal of Sound and Vibration 101, 511-521 (1985).

17. D. Brika, and A. Laneville, "Wake interference between two circular cylinders," J. Wind Eng. Ind. Aerodyn. 72, 61-70 (1997).

18. D. Brika, and A. Laneville, "The flow interaction between a stationary cylinder and a downstream flexible cylinder," J. Fluids Struct. 13, 579-606 (1999).

19. F. J. Huera-Huarte, and P. W. Bearman, "Vortex and wake-induced vibrations of a tandem arrangement of two flexible circular cylinders with near wake interference," Journal of Fluids and Structures 27, 193 - 211 (2011). 20. F. J. Huera-Huarte, and M. Gharib, "Vortex- and wake-induced vibrations of a tandem arrangment of two flexible circular cylinders with far wake interference," Journal of Fluids and Structures 27, 824 - 828 (2011).

21. B. S. Carmo, S. J. Sherwin, P. W. Bearman, and R. H. J. Willden, "Flow-induced vibration of a circular cylinder subjected to wake interference at low Reynolds number," Journal of Fluids and Structures 27, 503 - 522 (2011).

22. B. S. Carmo, G. R. S. Assi, and J. R. Meneghini, "Computational simulation of flow-induced vibation of a circular cylinder subjected to wake interference," Journal of Fluids and Structures 41, 99 - 108 (2013).

23. M. Zhao, "Flow induced vibration of two rigidly coupled circular cylinders in tandem and side-by-side arrangments at a low Reynolds number of 150," Physics of Fluids 25, 1236011 - 12360131 (2013). 
24. L. Ding, L. Zhang, C. Wu, E. S. Kim, and M. M. Bernitsas, "Numerical study on the effect of tandem spacing on flow-induced motions of two cylinders with passive turbulence control," Journal of Offshore Mechanics and Arctic Engineering 139, 0218011 - 0218018 (2017).

25. G. V. Papaioannou, D. K. P. Yue, M. S. Triantafyllou, and G. E. Karniadakis, "On the effect of spacing on the vortex-induced vibrations of two tandem cylinders," Journal of Fluids and Structures 24, 833 - 854 (2008).

26. T. K. Prasanth, and S. Mittal, "Flow-induced oscillation of two circular cylinders in tandem arrangement at low Re," Journal of Fluids and Structures 25, 1029 - 1048 (2009).

27. Y. Bao, C. Huang, D. Zhou, J. Tu, and Z. Han, "Two-degree-of-freedom flow-induced vibrations on isolated and tandem cylinders with varying natural frequency ratios," Journal of Fluids and Structures 35, 50 - 75 (2012). 28. H. C. Chen, C. R. Chen, and K. Huang, CFD simulation of vortex-induced and wake-induced vibrations of dual vertical risers (Anchorage, Alaska, USA, 2013).

29. L. M. González, A. Rodriguez, C. A. Garrido, J. C. Suarez, and F. J. Huera Huarte, CFD simualtion on the vortex-induced vibrations of a flexible cylinder with wake interference, OMAE2015-41128 (St. Johns, Canada, 2015).

30. F. J. Huera-Huarte, Z. A. Bangash, and L. M. González, Towing tank experiments on the vortex-induced vibrations of a flexible cylinder with wake interference, OMAE2014-23873 (San Francisco, California, USA, 2014).

31. ANSYS Inc., ANSYS Mechanical APDL Coupled-Field Analysis Guide (Canonsburg, USA, 2013).

32. ANSYS Inc., ANSYS CFX-Solver Theory Guide (Canonsburg, USA, 2013).

33. C. Rhie, and W. Chow, A numerical study of the turbulent flow past an isolated airfoil with trailing edge separation (AIAA, St. Louis, USA, 1982).

34. T. J. Barth, and D. C. Jespersen, The design and application of upwind schemes on unstructured meshes (AIAA, Reno, USA, 1989).

35. H. M. Hilber, T. J. R. Hughes, and R. L. Taylor, "Improved numerical dissipation for time integration algorithm in structural dynamics," Earthquake Engineering and Structural Dynamics 5, 283-292 (1977).

36. ANSYS Inc., ANSYS Mechanical APDL Element Reference (Canonsburg, USA, 2013).

37. M. Zhao, L. Cheng, H. An, and L. Lu, "Three-dimensional numerical simulation of vortex-induced vibration of an elastically mounted rigid circular cylinder in steady current," Journal of Fluids and Structures 50, 292 - 311 (2014).

38. E. Wang, and Q. Xiao, "Numerical simulation of vortex-induced vibration of a vertical riser in uniform and linearly sheared currents," Ocean Engineering 121, 492 - 515 (2016).

39. E. Wang, Q. Xiao, and A. Incecik, "Three-dimensional numerical simulation of two-degree-of-freedom VIV of a circular cylinder with varying natural frequency ratios at $\mathrm{Re}=500$," Journal of Fluids and Structures 73, 162 - 182 (2017).

40. C. Evangelinos, and G. E. Karniadakis, "Dynamics and flow structures in the turbulent of rigid and flexible cylinders subject to vortex-induced vibrations," Journal of Fluid Mechanics 400, 91 - 124 (1999).

41. C. Evangelinos, D. Lucor, and G. E. Karniadakis, "DNS-derived force distribution on flexible cylinders subject to vortex-induced vibration," Journal of Fluids and Structures 14, 429 - 440 (2000).

42. F. Xie, J. Deng, Q. Xiao, and Y. Zheng, "A numerical simulation of VIV on a flexible circular cylinder," Fluid Dynamics Research 44, 1 - 21 (2012). 
43. G. V. Papaioannou, D. K. P. Yue, M. S. Triantafyllou, and G. E. Karniadakis, "Three-dimensionality effect in flow around two tandem cylinders," Journal of Fluid Mechanics 558, 387 - 413 (2006).

44. T. Igarashi, "Characteristics of the flow aroudn two circular cylinders arranged in tandem (second report, unique flow phenomenon at small spacing)," Bulletin of the JSME 27, 2380 - 2387 (1984).

45. G. V. Papaioannou, "A Numerical Study of Flow-Structure Interactions with Application to Flow Past a Pair of Cylinders," PhD thesis (Massachusetts Institute of Technology, Massachusetts, USA, 2004).

46. G. Xu, and Y. Zhou, "Strouhal numbers in the wake of two inline cylinders," Experiments in Fluids 37, 248 256 (2004).

47. Y. Zhou, and M. W. Yiu, "Flow structure, momentum and heat transport in a two-tandem-cylinder wake," Journal of Fluid Mechanics 548, 17 - 48 (2006).

48. B. S. Carmo, J. R. Meneghini, and S. J. Sherwin, "Possible states in the flow around two circular cylinders in tandem with separations in the vicinity of the drag inversion spacing," Physics of Fluids 22, 1 - 7 (2010).

49. B. S. Carmo, J. R. Meneghini, and S. J. Sherwin, "Secondary instabilities in the flow around two circular cylinders in tandem " Journal of Fluid Mechanics 644, 395 - 431 (2010).

50. D. Sumner, "Two circular cylinders in cross-flow: A review," Journal of Fluids and Structures 26, 849 - 899 (2010).

51. E. Lehn, "VIV Suppression Tests on High L/D Flexible Cylinders," (Norwegian Marine Technology Research Institute, Trondheim, Norway, 2003).

52. M. Zhao, and L. Cheng, "Vortex-induced vibration of a circular cylinder of finite length," Physics of Fluids 26, 0151111 - 01511126 (2014).

53. M. Zhao, and L. Cheng, "Numerical simulation of two-degree-of-freedom vortex-induced vibration of a circular cylinder close to a plane boundary," Journal of Fluids and Structures 271097 - 1110 (2011).

54. F. J. Huera-Huarte, and P. W. Bearman, "Wake structures and vortex-induced vibrations of a long flexible cylinder - Part I: Dynamic response," Journal of Fluids and Structures 25, 969 - 990 (2009).

55. A. Pikovsky, M. Rosenblum, and J. Kurths, Synchronisation: A Universal Concept in Nonlinear Sciences (Cambridge University Press, New York, USA, 2001).

56. L. Ljungkrona, and B. Sundén, "Flow visualisation and surface pressure measurement on two tubes in an inline arrangement," Experimental Thermal and Fluid Science 6, 15 - 27 (1993).

57. R. Govardhan, and C. H. K. Williamson, "Modes of vortex formation and frequency response for a freelyvibrating cylinder," Journal of Fluid Mechanics 420, 85 - 130 (2000).

58. A. Laneville, and D. Brika, "The fluid and mechanical coupling between two circular cylinders in tandem arrangement," J. Fluids Struct. 13, (1999).

59. F. S. Hover, A. H. Techet, and M. S. Triantafyllou, "Forces on oscillating uniform and tapered cylinders in crossflow," Journal of Fluid Mechanics 363, 97 - 114 (1998).

60. F. S. Hover, J. T. Davis, and M. S. Triantafyllou, "Three-dimensional mode transition in vortex-induced vibrations of a circular cylinder," European Journal of Mechanics-B/Fluids 23, 29 - 40 (2004).

61. D. Lucor, J. Foo, and G. E. Karniadakis, Correlation length and force phasing of a rigid cylinder subject to VIV (Springer Dordrecht, 2003).

62. D. Lucor, J. Foo, and G. E. Karniadakis, "Vortex mode selection of a rigid cylinder subject to VIV at low mass-damping," Journal of Fluids and Structures 20, 483 - 503 (2005). 
63. J. Wu, L. W. Welch, M. C. Welsh, J. Sheridan, and W. G.J., "Spanwise wake structures of a circular cylinder and two circular cylinders in tandem," Experimental Thermal and Fluid Science 9, 299 - 308 (1994).

64. J. Jeong, and F. Hussain, "On the identification of a vortex," Journal of Fluid Mechanics 285, 69 - 94 (1995).

65. C. H. K. Williamson, and A. Roshko, "Vortex formation in the wake of an oscillating cylinder," Journal of Fluids and Structures 2, 355 - 381 (1988).

66. D. Brika, and A. Laneville, "Vortex-induced vibrations of a long flexible circular cylinder," Journal of Fluid Mechanics 250, 481 - 508 (1993).

67. D. Brika, and A. Laneville, "An experimental study of the aeolian vibrations of a flexible circular cylinder at different incidences," Journal of Fluids and Structures 9, 371 - 391 (1995).

68. N. Jauvtis, and C. H. K. Williamson, "The effect of two degrees of freedom on vortex-induced vibration at low mass and damping," Journal of Fluid Mechanics 509, 23 - 62 (2004).

69. O. M. Griffin, and S. E. Ramberg, "The vortex street wakes of vibrating cylinders," Journal of Fluid Mechanics 66, 553 - 576 (1974).

70. R. Zdera, Ö. F. Turan, and D. G. Havard, "Towards understanding galloping: Near-wake sutdy of oscillating smooth and stranded circular cylinders in forced motion," Experimental Thermal and Fluid Science 10, 28 - 43 (1995).

71. S. P. Singh, and S. Mittal, "Vortex-induced oscillations at low Reynolds numbers: Hysteresis and vortex shedding modes," Journal of Fluids and Structures 20, 1085 - 1104 (2005).

72. E. D. Gedikli, and J. M. Dahl, Mode shape vibration for a low-mode number flexible cylinder subject to vortexinduced vibrations (San Francisco, USA, 2014). 
Table 1. CFD mesh dependency test results.

\begin{tabular}{cccccccc}
\hline CFDM & $N_{c}$ & $\Delta r / D$ & $\Delta z / D$ & $A_{y} / D$ & $C_{\text {Dmean }}$ & $C_{\text {Drms }}$ & $C_{\text {Lrms }}$ \\
\hline 1 & 80 & 0.002 & 0.2 & 0.7443 & 1.5975 & 0.2501 & 0.1717 \\
2 & 120 & 0.001 & 0.1 & 0.7480 & 1.6127 & 0.2449 & 0.1661 \\
3 & 160 & 0.0005 & 0.05 & 0.7524 & 1.6039 & 0.2482 & 0.1638 \\
\hline
\end{tabular}

Table 2. FEA mesh dependency test results.

\begin{tabular}{cccccc}
\hline FEAM & $N_{z}$ & $A_{y} / D$ & $C_{\text {Dmean }}$ & $C_{\text {Drms }}$ & $C_{\text {Lrms }}$ \\
\hline 1 & 15 & 0.7515 & 1.5964 & 0.2421 & 0.1673 \\
2 & 30 & 0.7480 & 1.6127 & 0.2449 & 0.1661 \\
3 & 60 & 0.7480 & 1.6076 & 0.2453 & 0.1652 \\
\hline
\end{tabular}




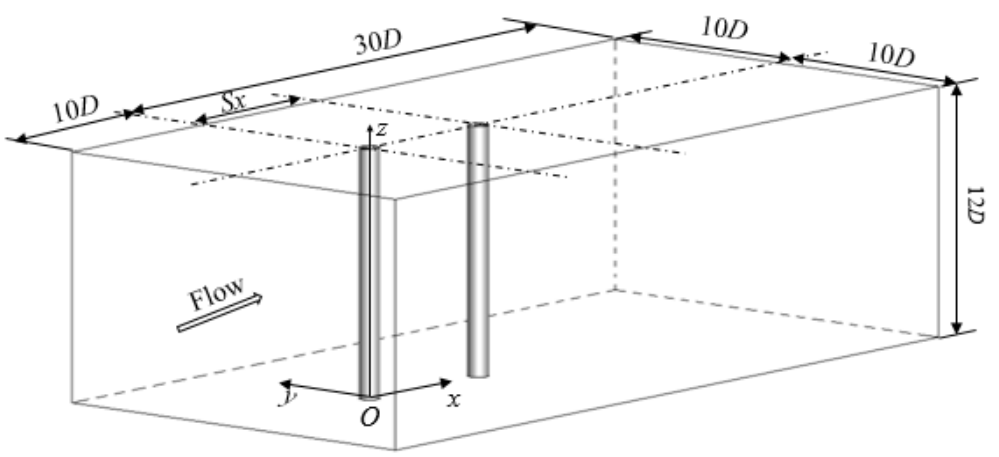

(a)

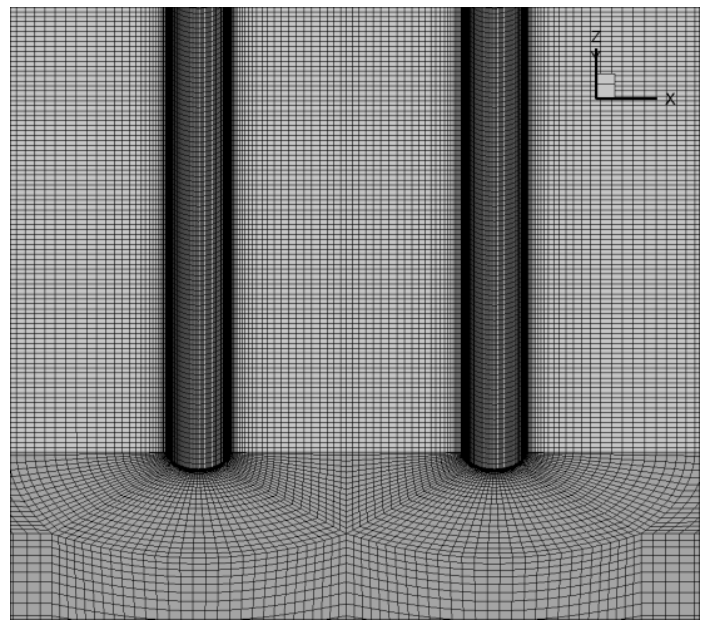

(b)

FIG. 1. (a) Computational domain and (b) computational mesh in the CFD simulation.

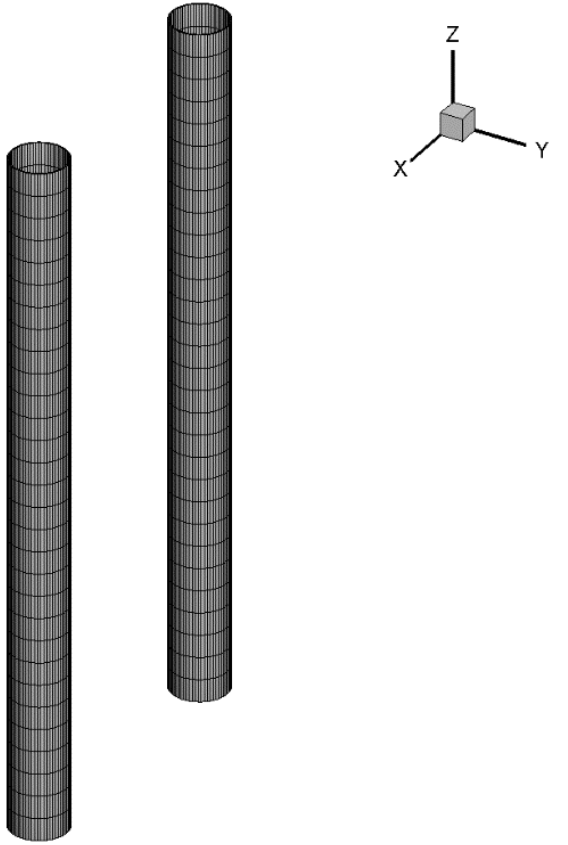

(a)

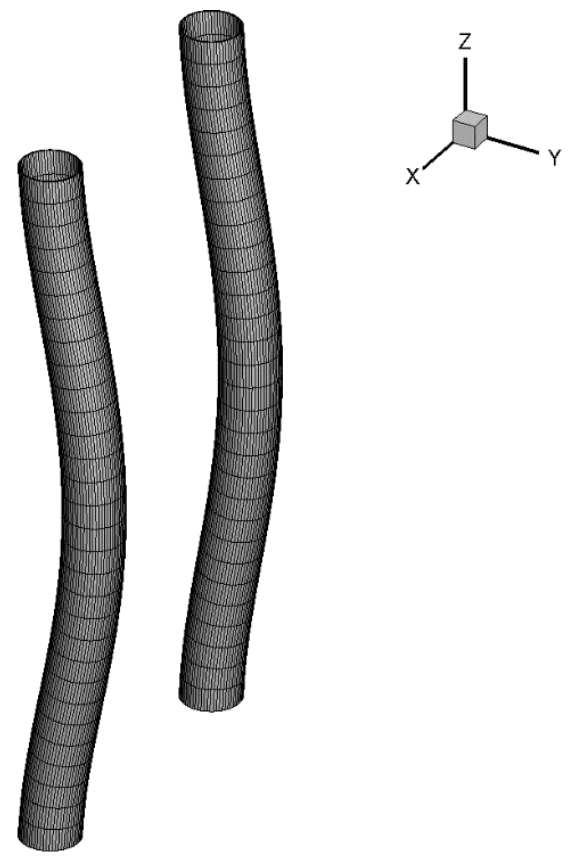

(b)

FIG. 2. Computational mesh in the FEA simulation at $S_{x} / D=5$ : (a) initial configuration and (b) mesh after deflections. 


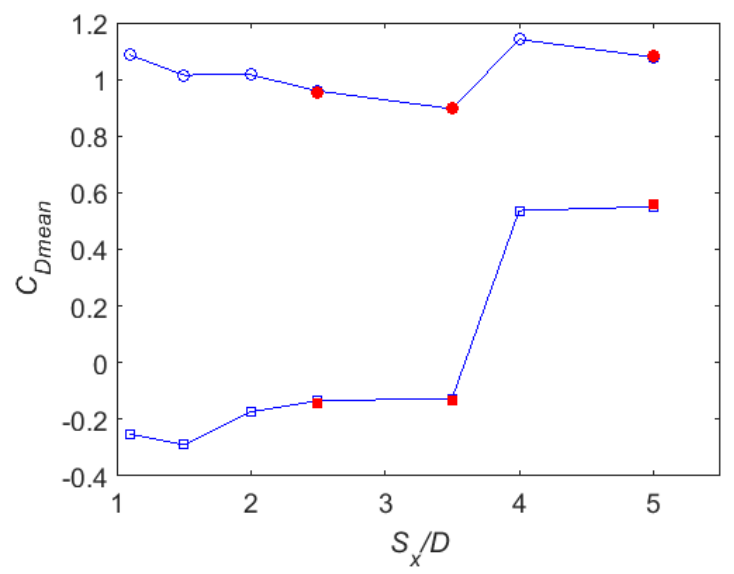

FIG. 3. Comparison of spanwise mean drag coefficients of two tandem stationary cylinders at $\operatorname{Re}=500$ : —०—, upstream cylinder (Papaioannou, et al., 2006); ———, downstream cylinder (Papaioannou, et al., 2006); •, upstream cylinder (present simulation); $\mathbf{n}$, downstream cylinder (present simulation).

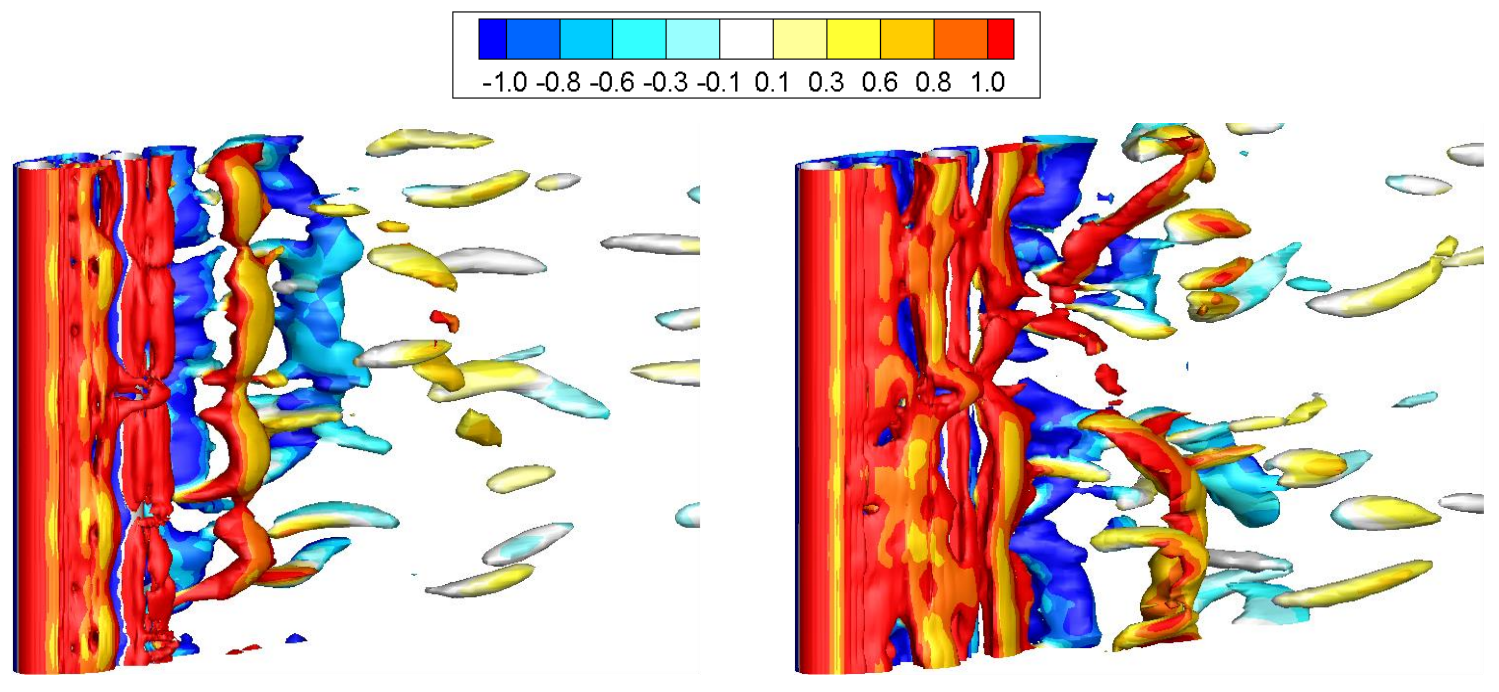

(a)

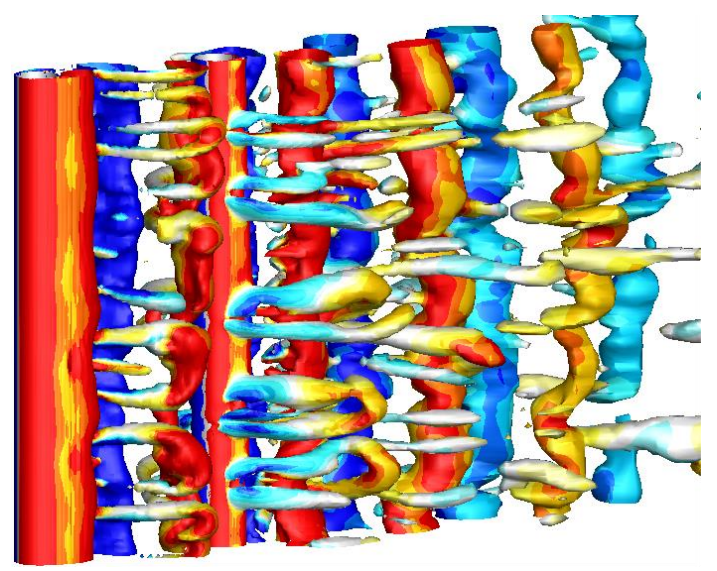

(c)

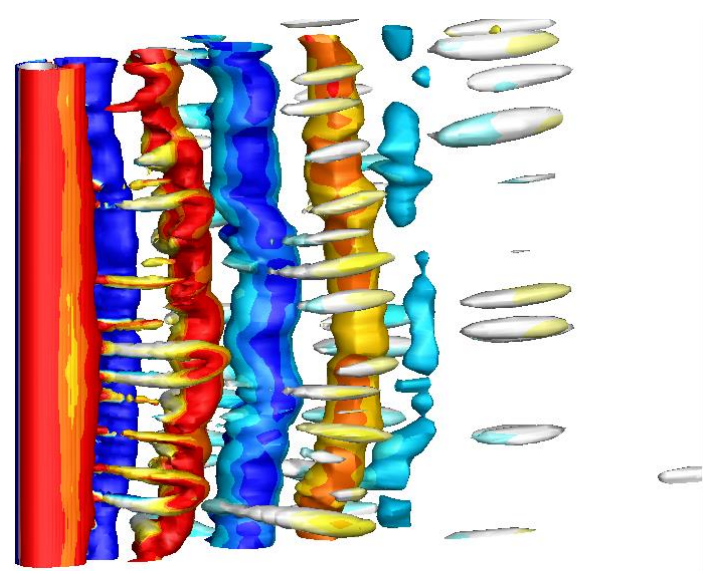

(d)

FIG. 4. Iso-surfaces of the eigenvalue $\lambda_{2}=-0.1$ with the contours of the spanwise vorticity $\omega_{z}$ on the iso-surfaces for stationary cylinders: (a) $S_{x} / D=2.5$, (b) $S_{x} / D=3.5$, (c) $S_{x} / D=5$ and (d) single cylinder. 


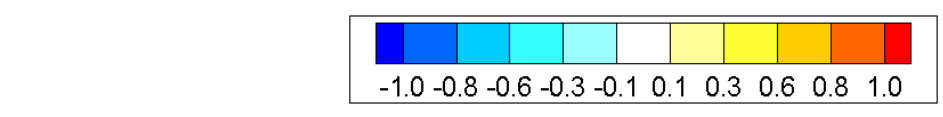

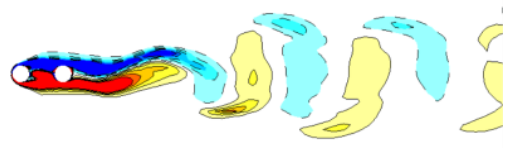

(a)

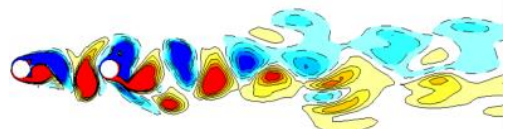

(c)

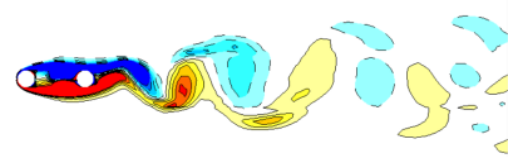

(b)

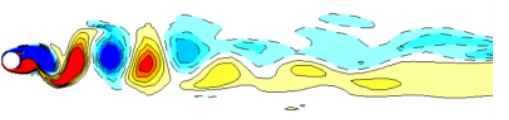

(d)

FIG. 5. Spanwise vorticity contours $\omega_{z}$ for stationary cylinders at $z / L=0.5$ : (a) $S_{x} / D=2.5$, (b) $S_{x} / D=3.5$, (c) $S_{x} / D=5$ and (d) single cylinder.

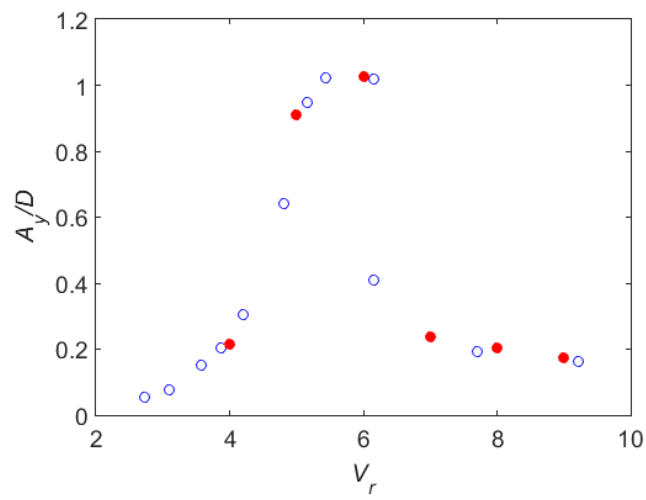

FIG. 6. Comparison of vibration amplitudes of a single flexible cylinder with $m^{*}=10, L / D=12$ at $\operatorname{Re}=1000$ : $\circ$, Xie et al. (2012); •, present simulation. 

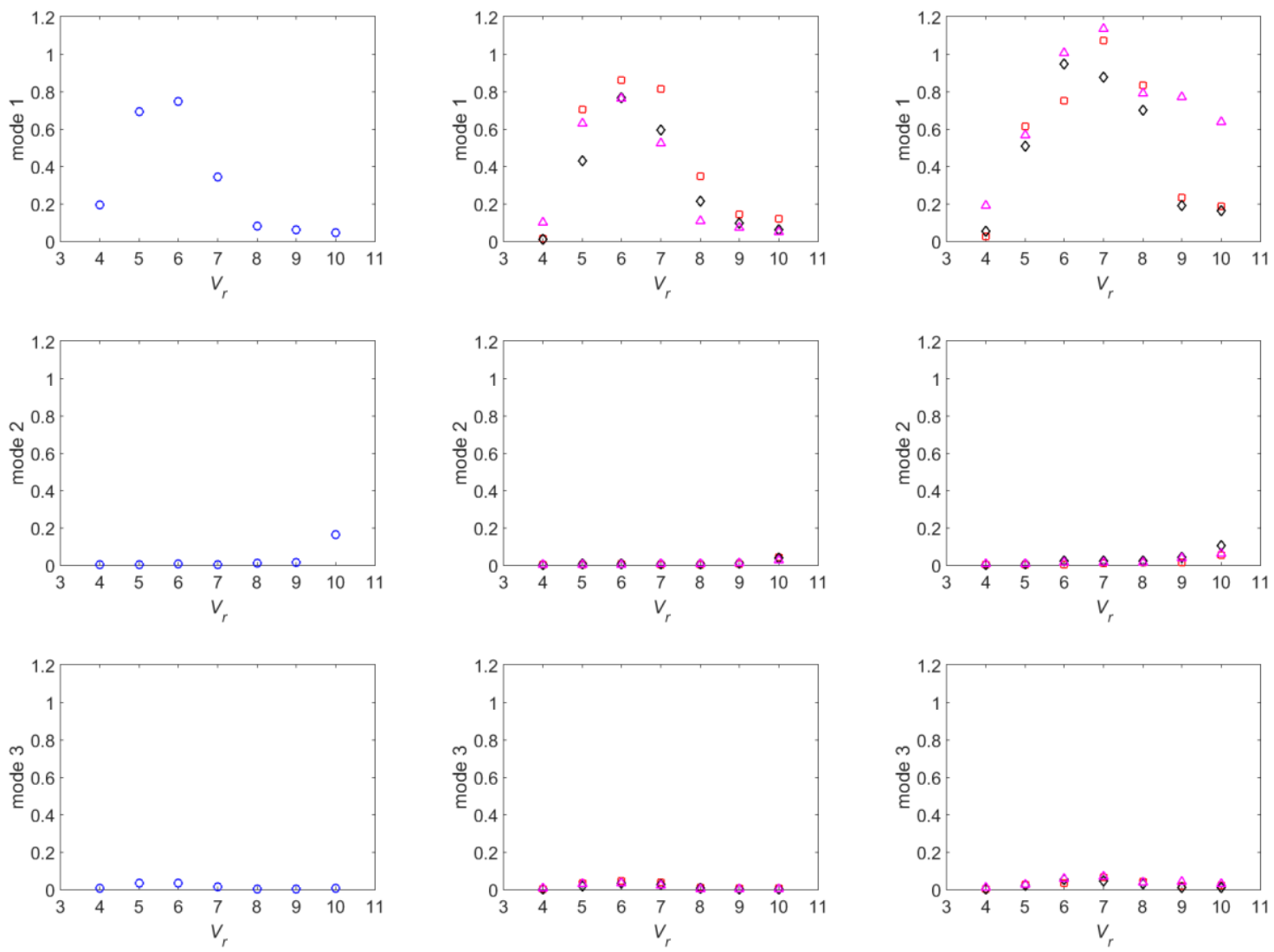

(a)

(b)

(c)

FIG. 7. Variation of modal amplitudes with $V_{r}$ : (a) single flexible cylinder, (b) upstream cylinder and (c) downstream cylinder. ॰, single flexible cylinder; $\square$, tandem, $S_{x} / D=2.5 ; \diamond$, tandem, $S_{x} / D=3.5 ; \Delta$, tandem, $S_{x} / D=5$. 


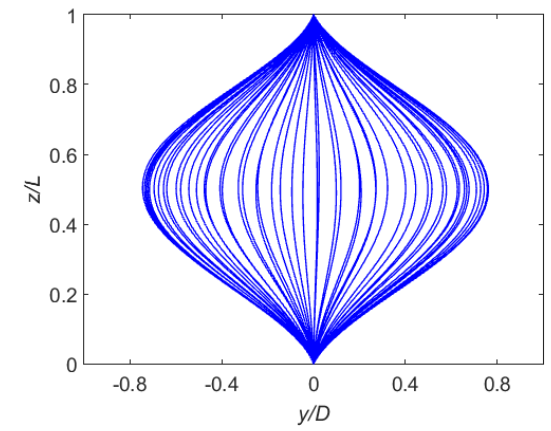

(a)

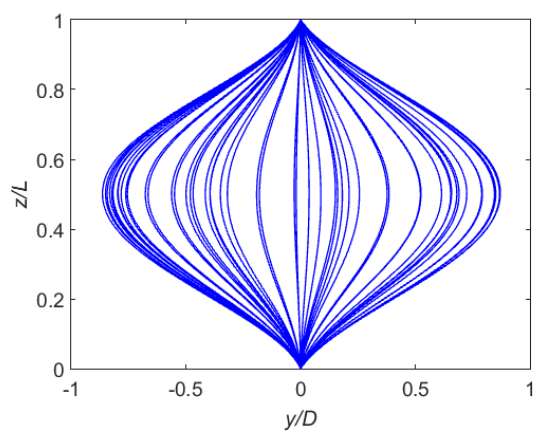

(b)

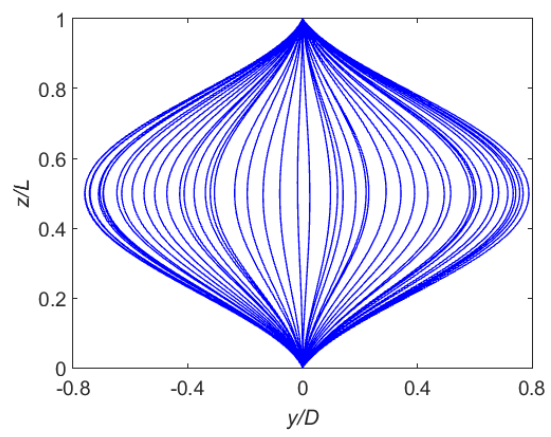

(d)

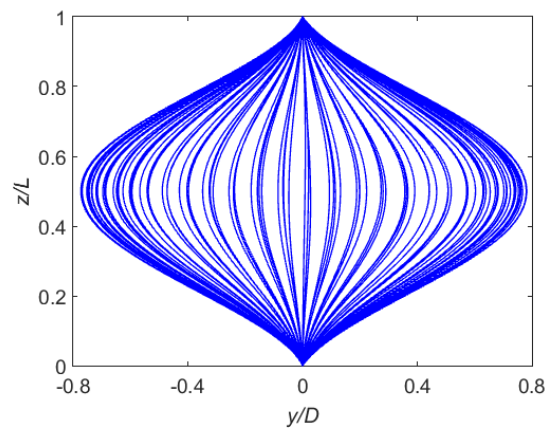

(f)

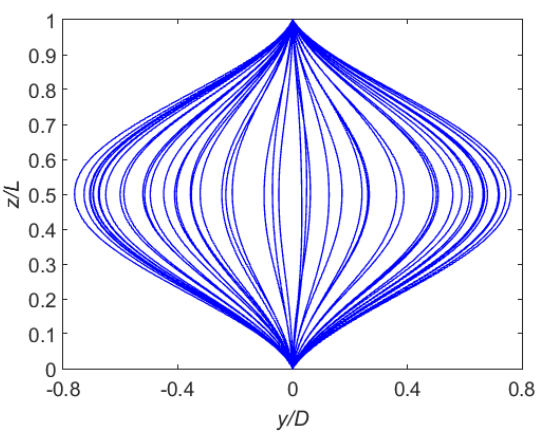

(c)

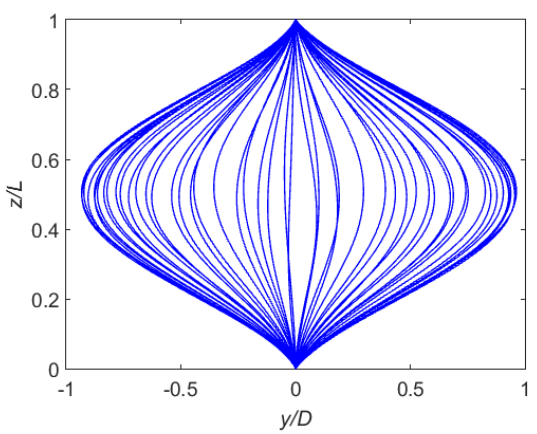

(e)

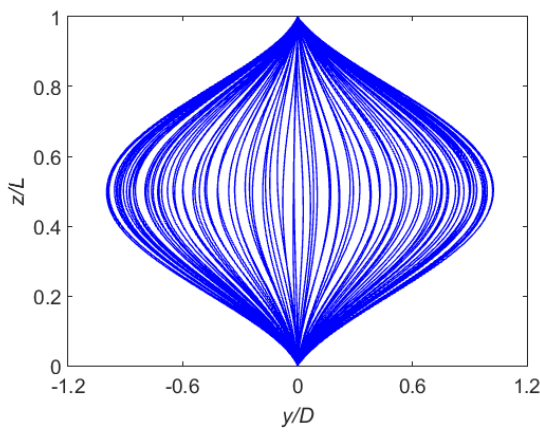

(g)

FIG. 8. Instantaneous nondimensional deflections of the flexible cylinders at $V_{r}=6$ : (a) single flexible cylinder, (b) upstream cylinder, $S_{x} / D=2.5$, (c) downstream cylinder, $S_{x} / D=2.5$, (d) upstream cylinder, $S_{x} / D=3.5$, (e) downstream cylinder, $S_{x} / D=$ 3.5, (f) upstream cylinder, $S_{x} / D=5$ and (g) downstream cylinder, $S_{x} / D=5$. 


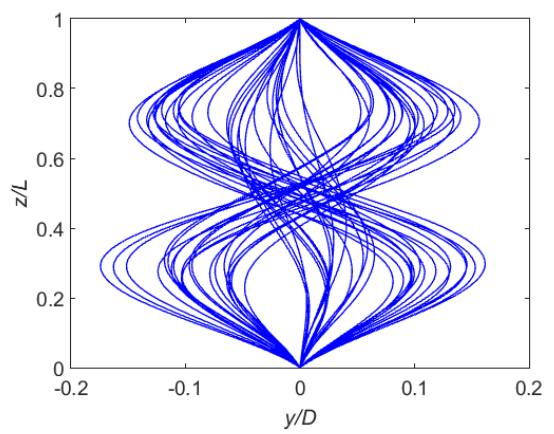

(a)

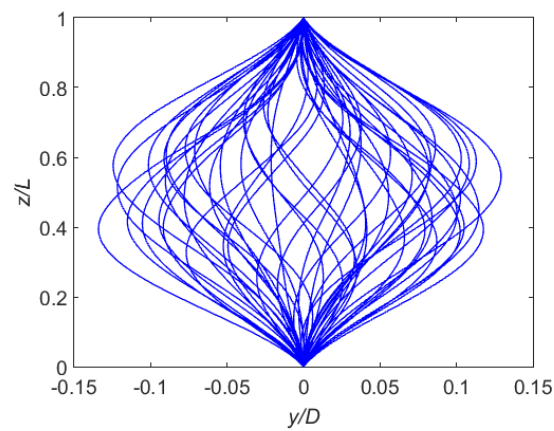

(b)

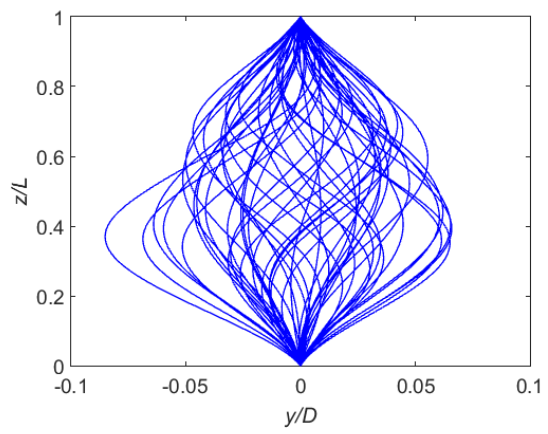

(d)

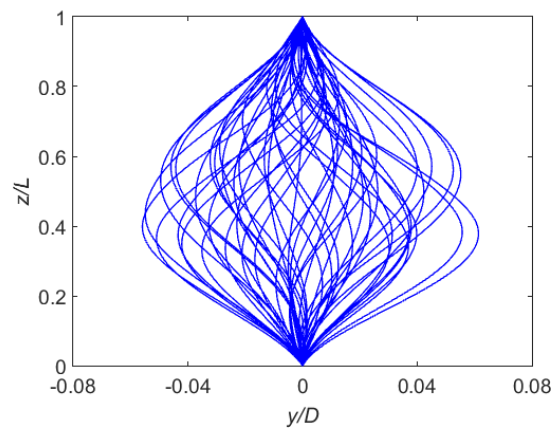

(f)

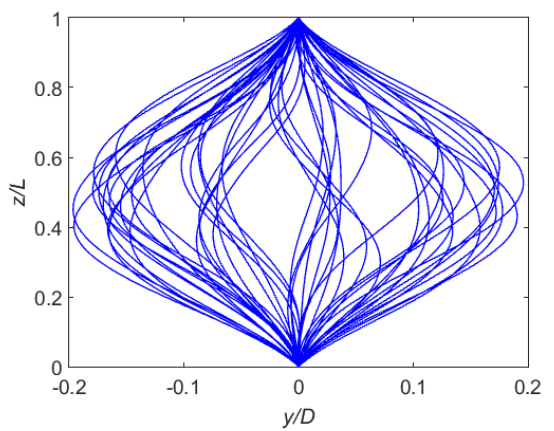

(c)

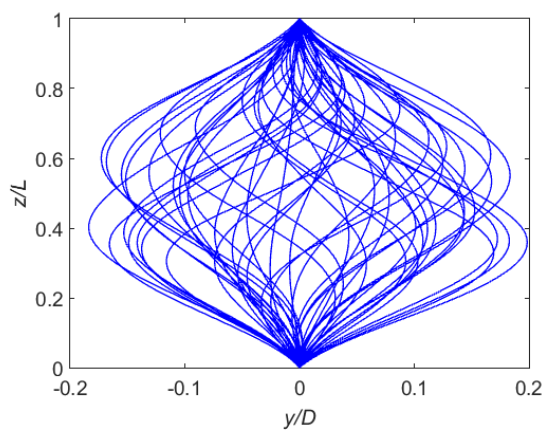

(e)

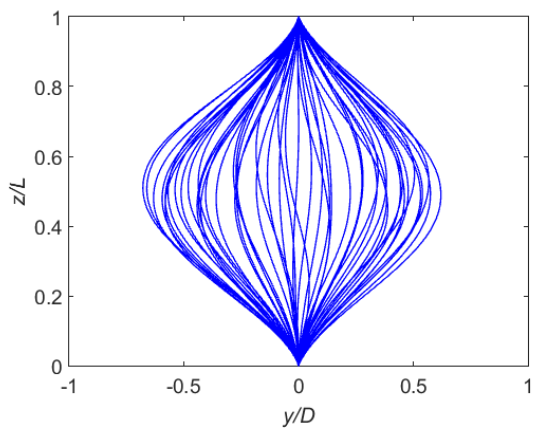

(g)

FIG. 9. Instantaneous nondimensional deflections of the flexible cylinders at $V_{r}=10$ : (a) single flexible cylinder, (b) upstream cylinder, $S_{x} / D=2.5$, (c) downstream cylinder, $S_{x} / D=2.5$, (d) upstream cylinder, $S_{x} / D=3.5$, (e) downstream cylinder, $S_{x} / D=3.5$, (f) upstream cylinder, $S_{x} / D=5$ and (g) downstream cylinder, $S_{x} / D=5$. 
$z / L=0.9$

$z / L=0.7$

$z / L=0.6$

$z / L=0.5$

$z / L=0.4$

$z / L=0.3$

$z / L=0.2$

$z / L=0.1$

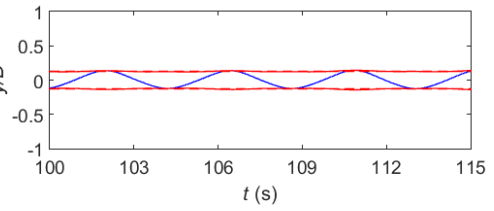

(a)
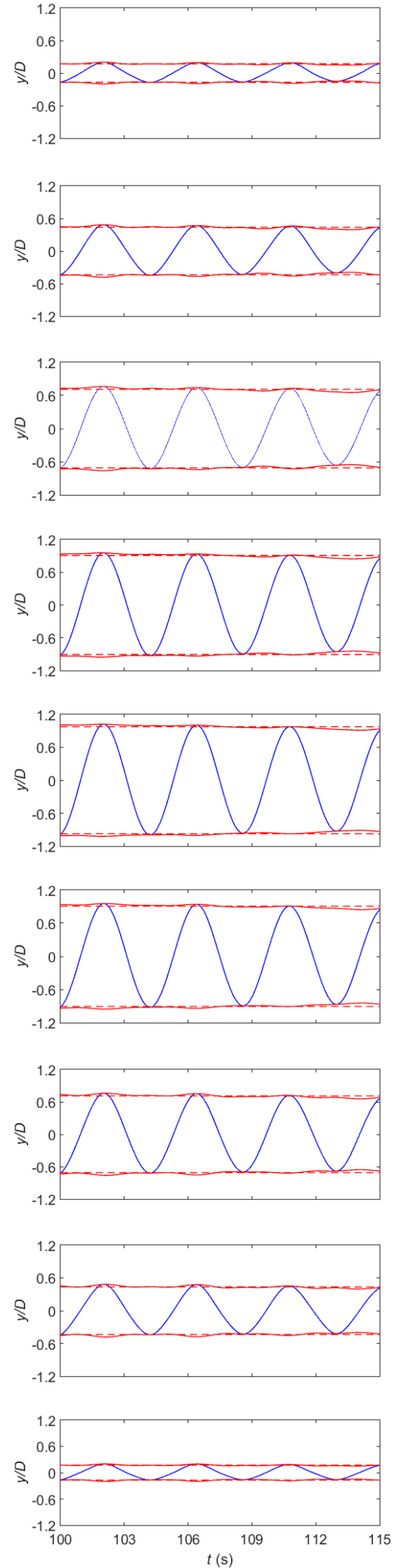

(b)

FIG. 10. Displacement time histories on different cross-sections along two tandem flexible cylinders at $V_{r}=6$ and $S_{x} / D=5$ : (a) upstream cylinder and (b) downstream cylinder. - , displacement signals; - , envelopes of the signals; ---, mean values of the envelopes. 


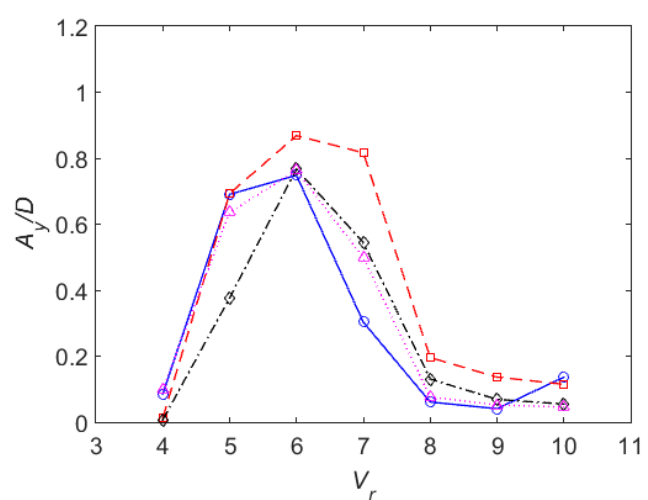

(a)

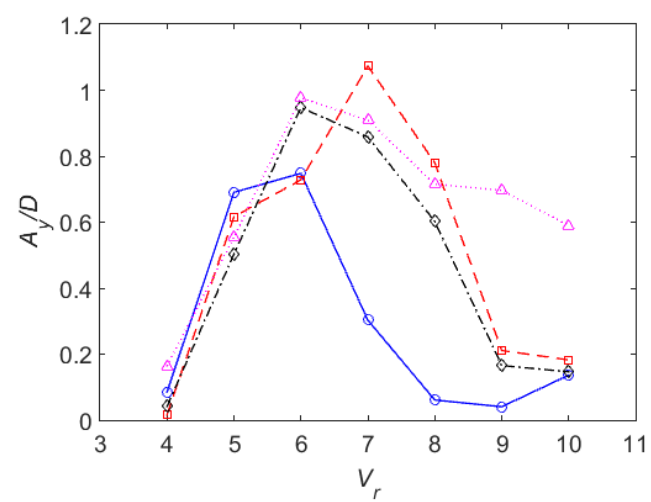

(b)

FIG. 11. Variation of the vibration amplitudes with $V_{r}$ for flexible cylinders: (a) upstream cylinder and (b) downstream

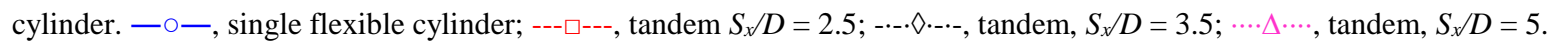

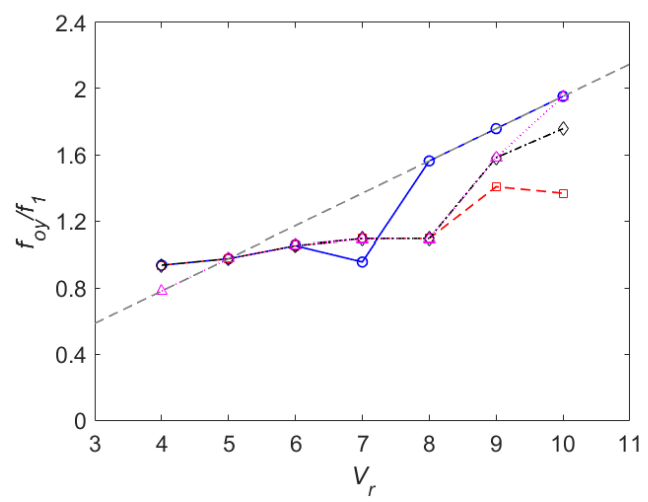

(a)

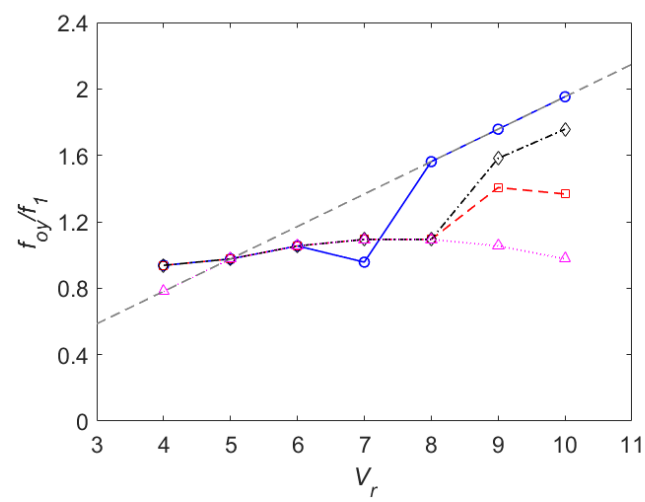

(b)

FIG. 12. Variation of oscillation frequencies with $V_{r}$ for flexible cylinders: (a) upstream cylinder and (b) downstream cylinder. -----, $\mathrm{St}=0.2 ;-\circ-$, single flexible cylinder; ---口---, tandem $S_{x} / D=2.5 ;-\cdot-\bullet \cdot-\cdot-$, tandem, $S_{x} / D=3.5 ; \cdots \Delta \cdots \cdot$ tandem, $S_{x} / D=5$.

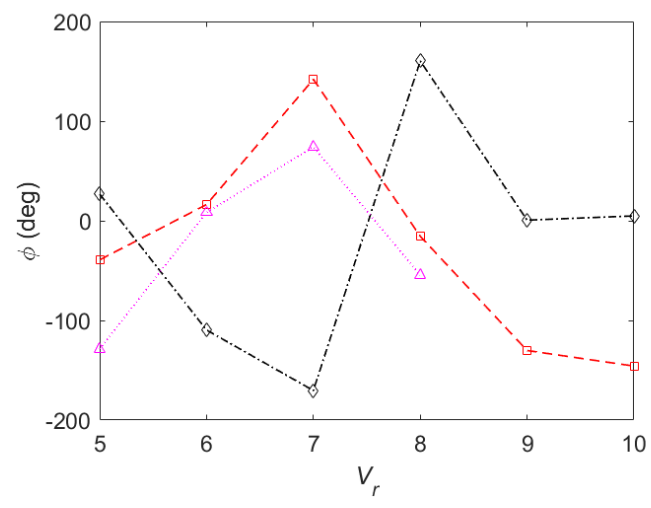

FIG. 13. Variation of phase differences $\phi$ between the cross-flow displacements at $z / L=0.5$ of the two tandem flexible cylinders in the time range of $t=100-115 \mathrm{~s}$ with $V_{r}:---\square---$, tandem $S_{x} / D=2.5 ;-\cdot-\diamond \cdot-\cdot-$, tandem, $S_{x} / D=3.5 ; \cdots \Delta \cdots$, tandem, $S_{x} / D=5$. 


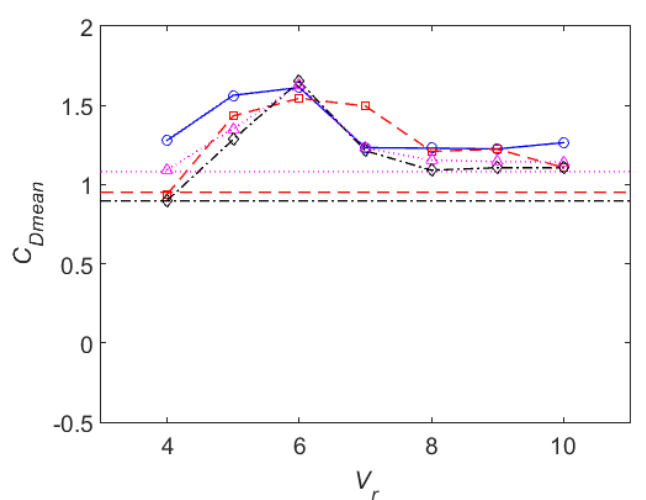

(a)

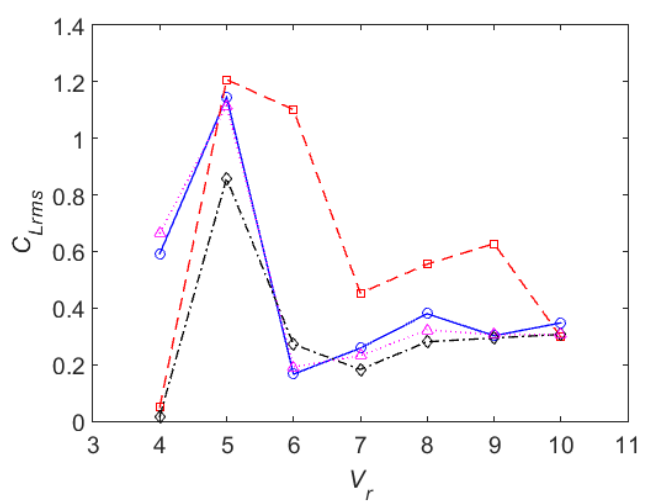

(c)

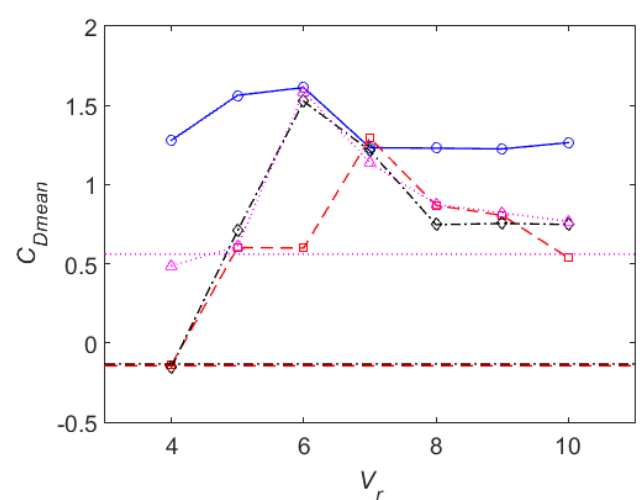

(b)

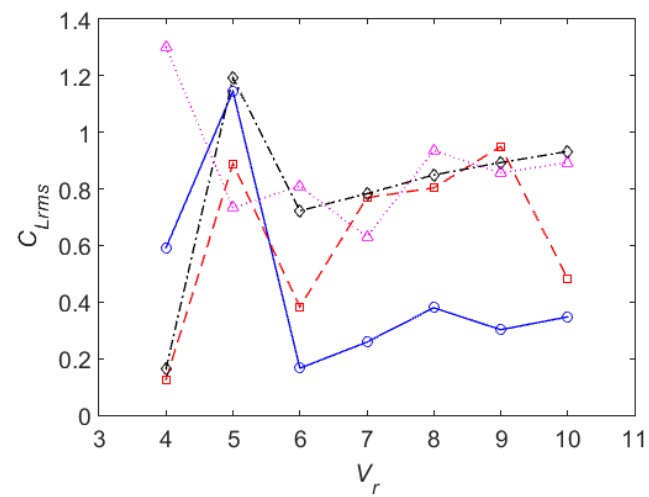

(d)

FIG. 14. Variation of total force coefficients with $V_{r}$ for flexible cylinders: (a) $C_{D \text { mean }}$ of the upstream cylinder, (b) $C_{D m e a n}$ of the downstream cylinder,.(c) $C_{\text {Lrms }}$ of the upstream cylinder and (d) $C_{\text {Lrms }}$ of the downstream cylinder. — —, single flexible cylinder; ---口---, tandem $S_{x} / D=2.5 ;-\cdot-\diamond \cdot \cdots \cdot-$, tandem, $S_{x} / D=3.5 ; \cdots \Delta \cdots$, tandem, $S_{x} / D=5$.

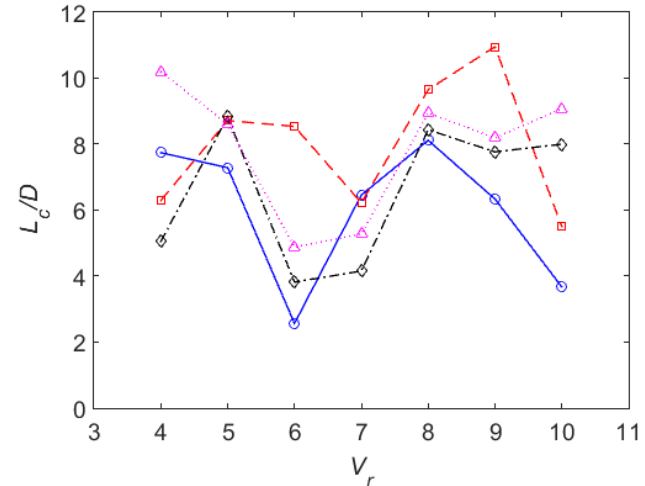

(a)

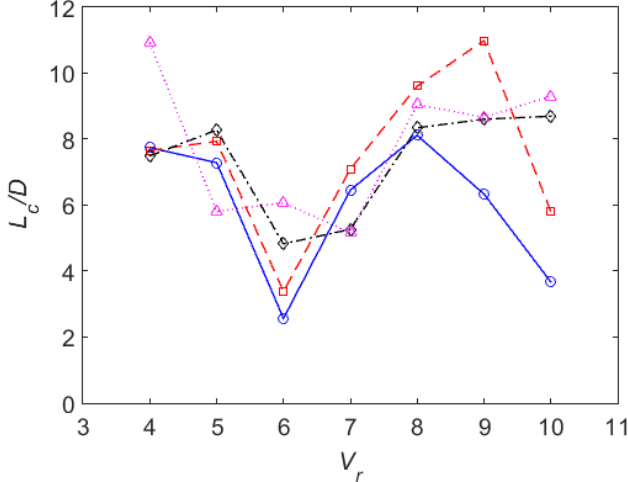

(b)

FIG. 15. Variation of correlation lengths with $V_{r}$ for flexible cylinders: (a) upstream cylinder and (b) downstream cylinder. ———, single flexible cylinder; ---口---, tandem $S_{x} / D=2.5 ;-\cdots \bullet \cdot \cdots \cdot-$, tandem, $S_{x} / D=3.5 ; \cdots \triangle \cdots$, tandem, $S_{x} / D=5$. 
$V_{r}=5$
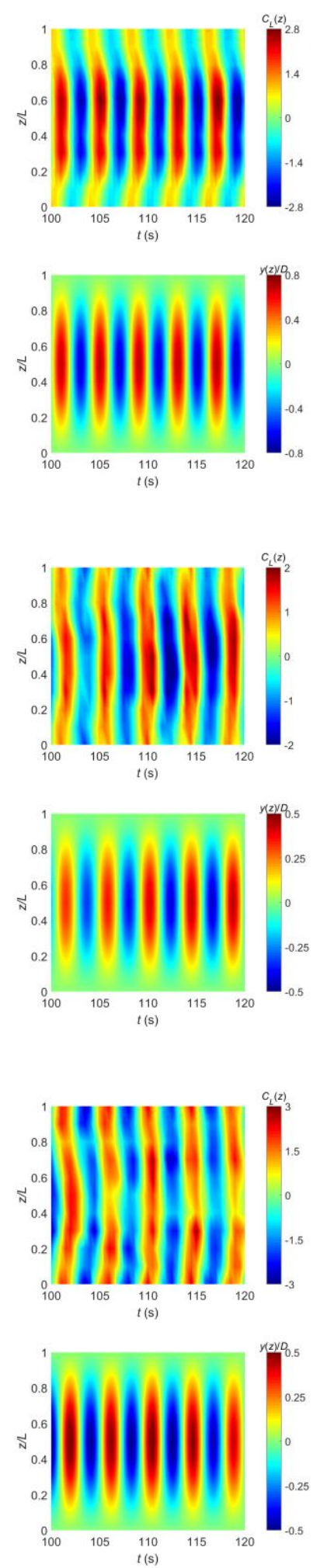

$V_{r}=6$
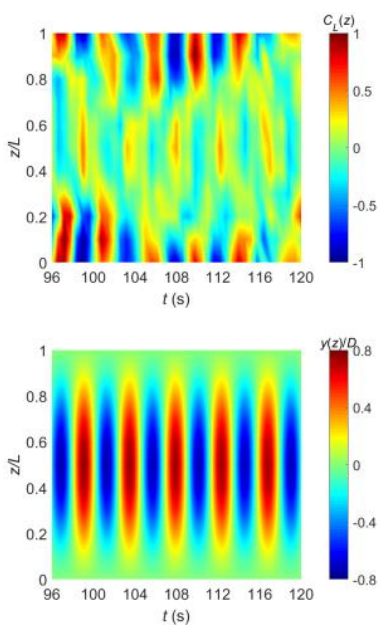

(a)
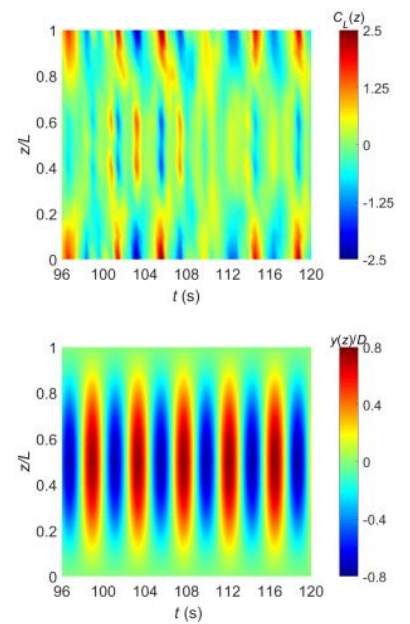

(b)
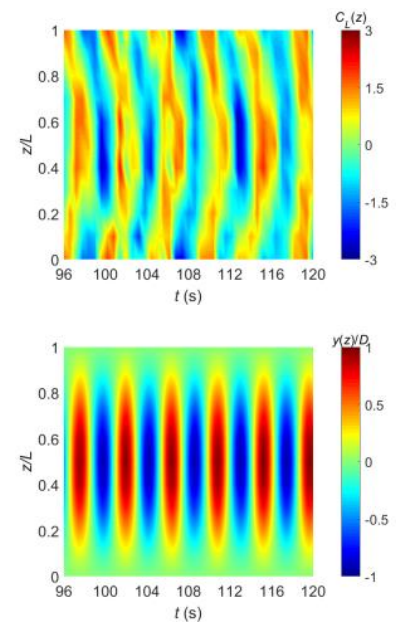

$V_{r}=8$
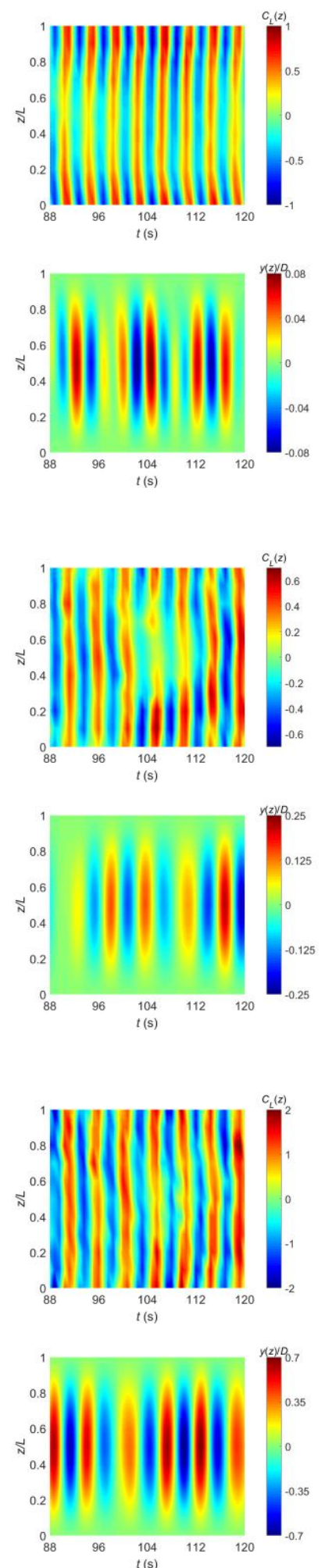

(c)

FIG. 16. Contours of sectional lift coefficients and sectional displacements of flexible cylinders: (a) single flexible cylinder, (b) upstream cylinder at $S_{x} / D=3.5$ and (c). downstream cylinder at $S_{x} / D=3.5$. 


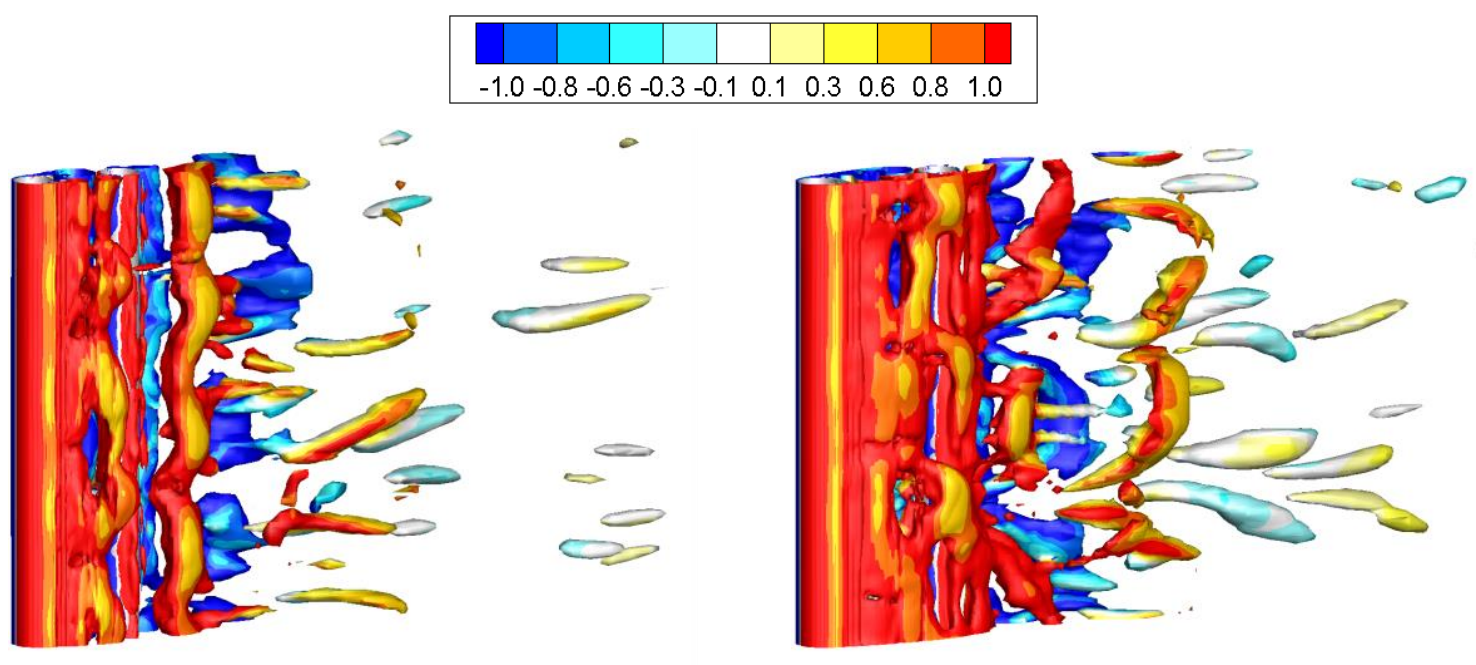

(a)

(b)
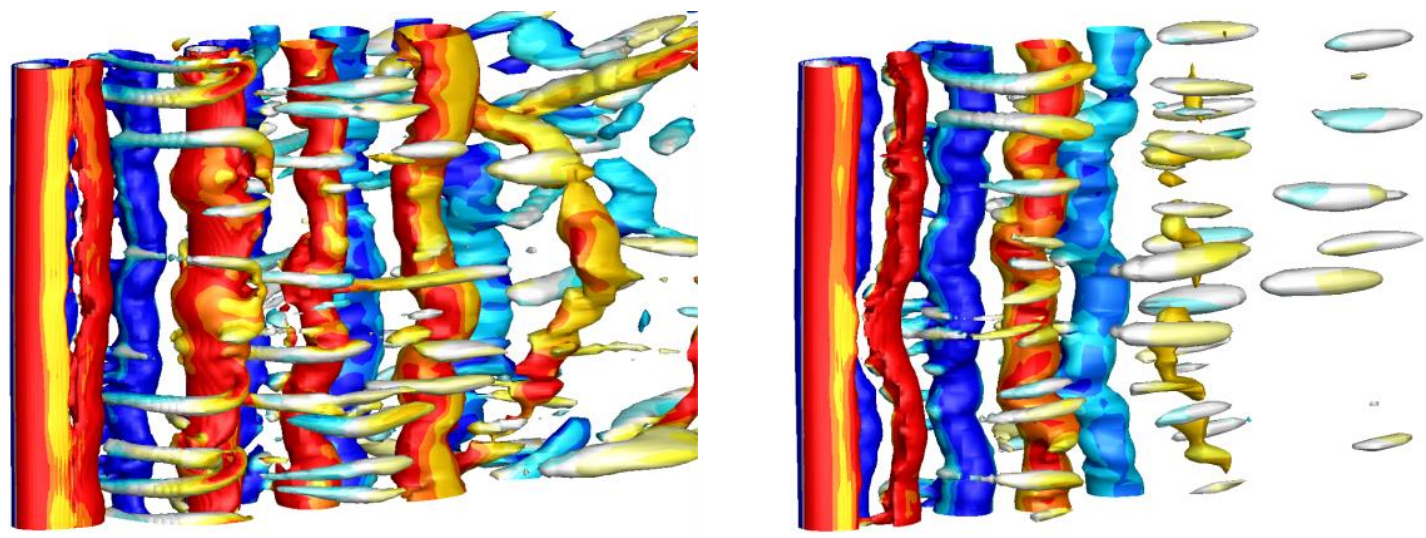

(c)

(d)

FIG. 17. Iso-surfaces of the eigenvalue $\lambda_{2}=-0.1$ with the contours of the spanwise vorticity $\omega_{z}$ on the iso-surfaces for flexible cylinders at $V_{r}=4$ : (a) $S_{x} / D=2.5$, (b) $S_{x} / D=3.5$, (c) $S_{x} / D=5$ and (d) single flexible cylinder. 


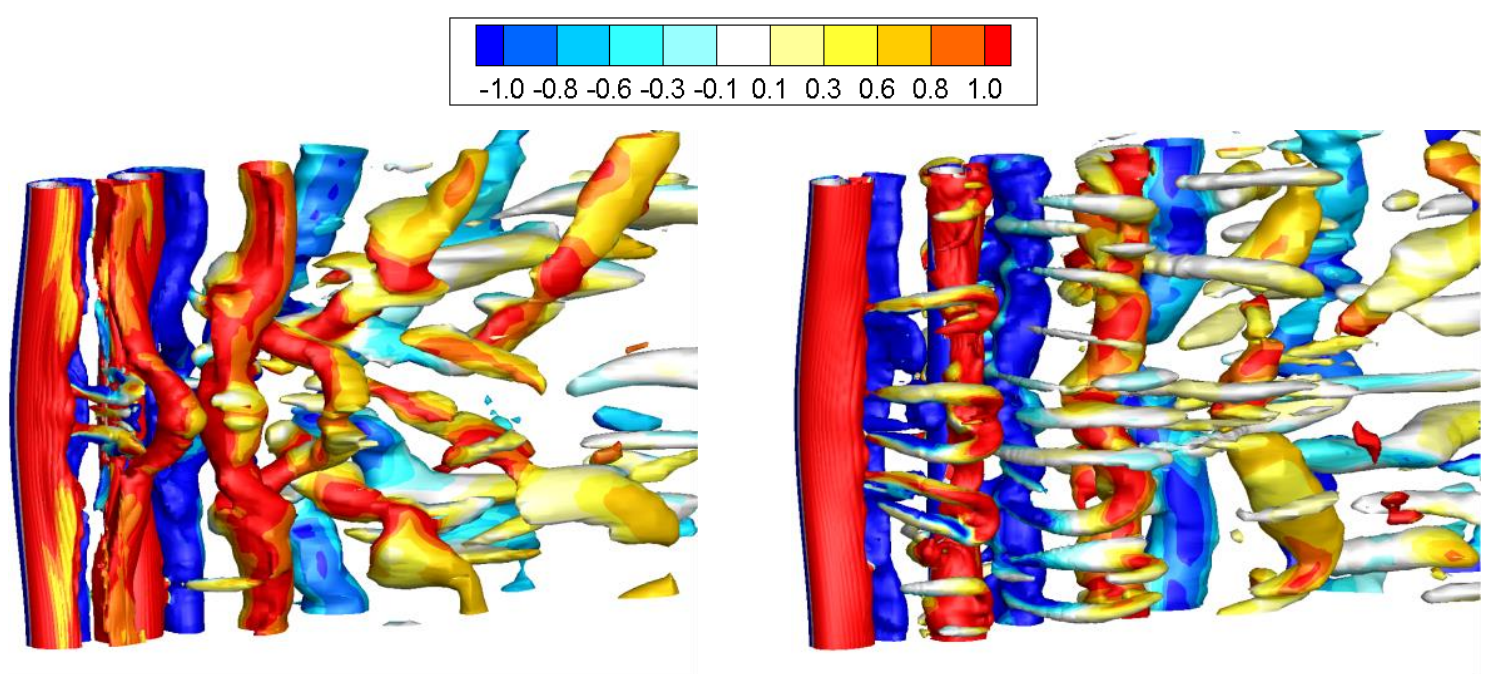

(a)

(b)

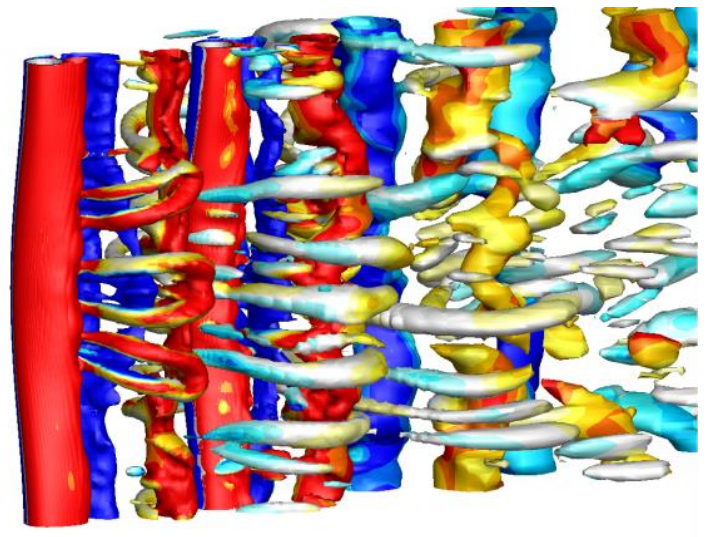

(c)

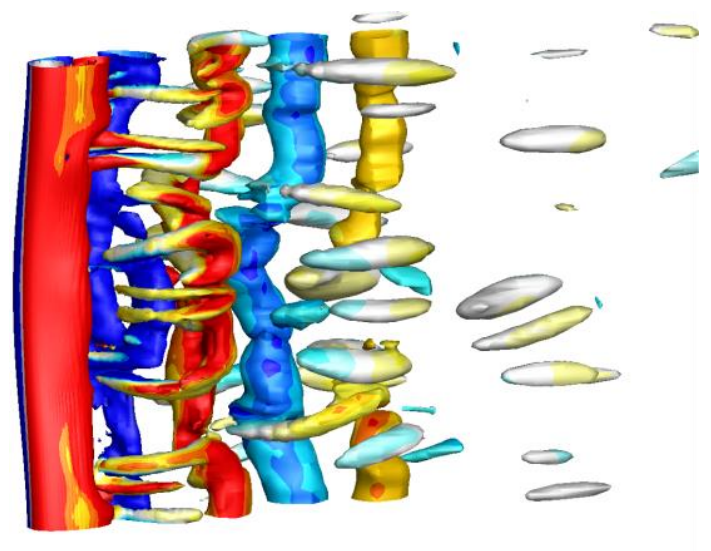

(d)

FIG. 18. Iso-surfaces of the eigenvalue $\lambda_{2}=-0.1$ with the contours of the spanwise vorticity $\omega_{z}$ on the iso-surfaces for flexible cylinders at $V_{r}=6$ : (a) $S_{x} / D=2.5$, (b) $S_{x} / D=3.5$, (c) $S_{x} / D=5$ and (d) single flexible cylinder. 


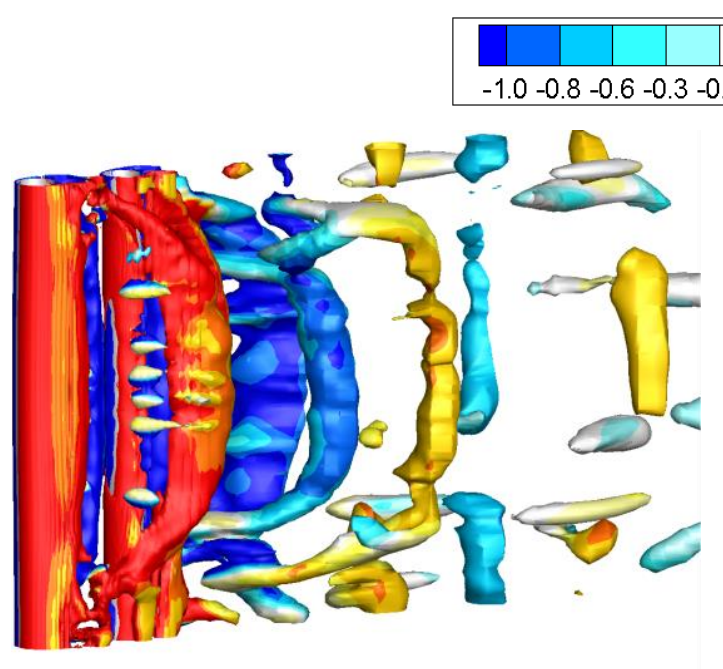

(a)

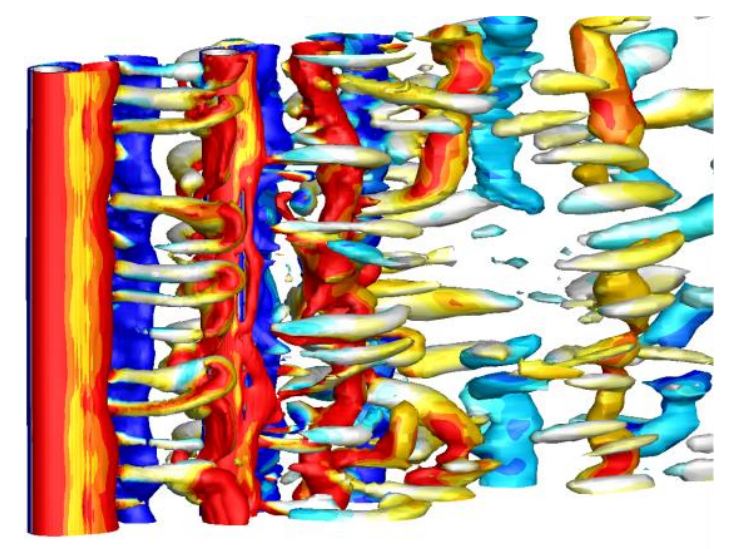

(c)

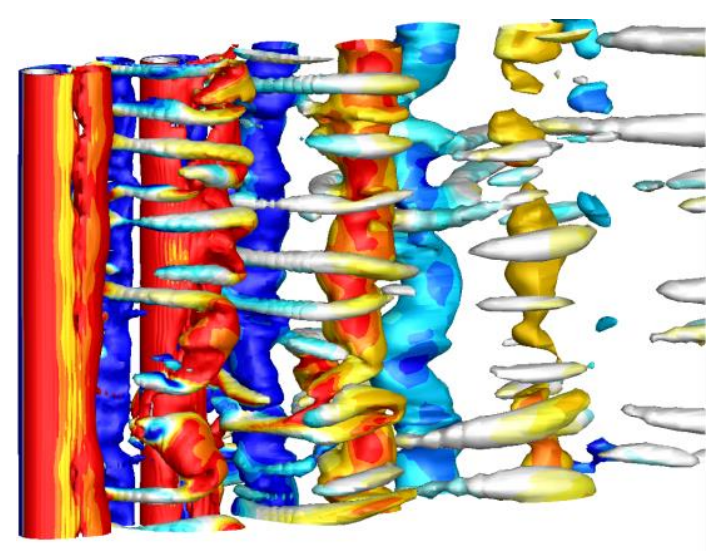

(b)

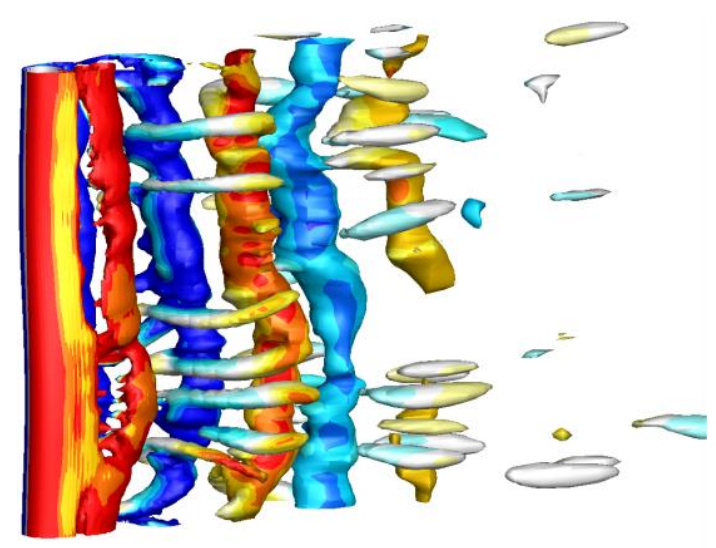

(d)

FIG. 19. Iso-surfaces of the eigenvalue $\lambda_{2}=-0.1$ with the contours of the spanwise vorticity $\omega_{z}$ on the iso-surfaces for flexible cylinders at $V_{r}=10$ : (a) $S_{x} / D=2.5$, (b) $S_{x} / D=3.5$, (c) $S_{x} / D=5$ and (d) single flexible cylinder. 


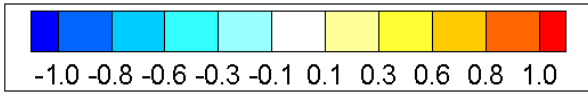

$z / L=0.25$

$z / L=0.5$

$z / L=0.75$
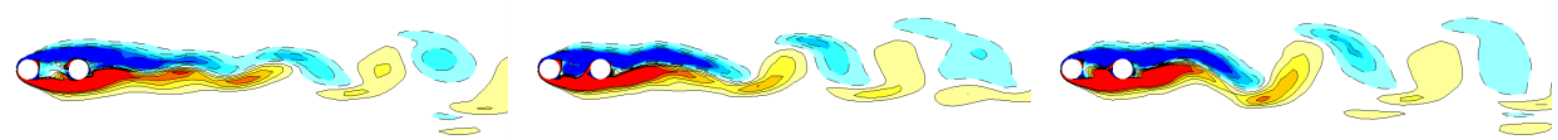

(a)
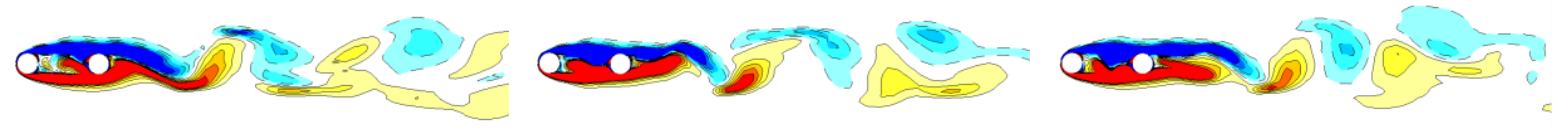

(b)
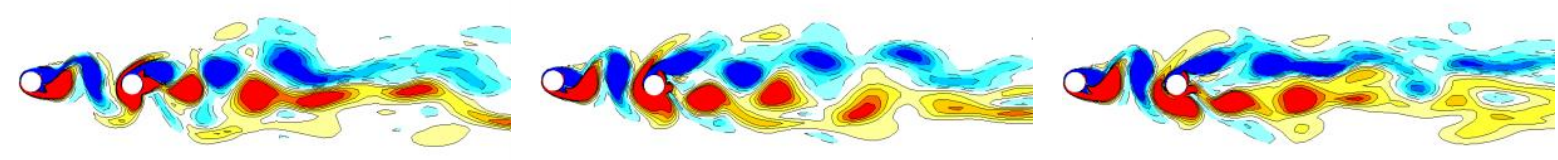

(c)
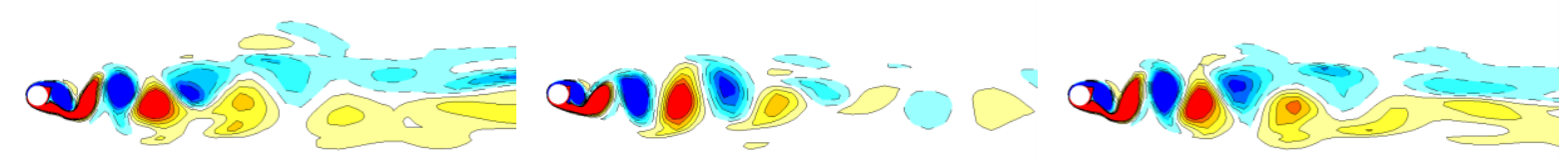

(d)

FIG. 20. Contours of spanwise vorticity $\omega_{z}$ on three different cross sections along the flexible cylinders at $V_{r}=4:$ (a) $S_{x} / D=$ 2.5, (b) $S_{x} / D=3.5$, (c) $S_{x} / D=5$ and (d) single flexible cylinder. 


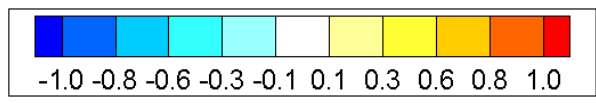

$z / L=0.25$

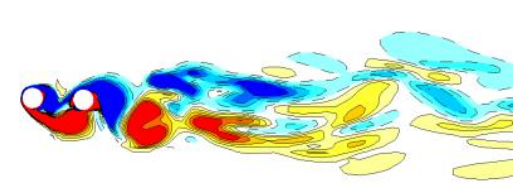

$z / L=0.5$

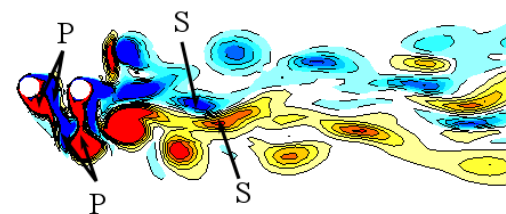

(a)
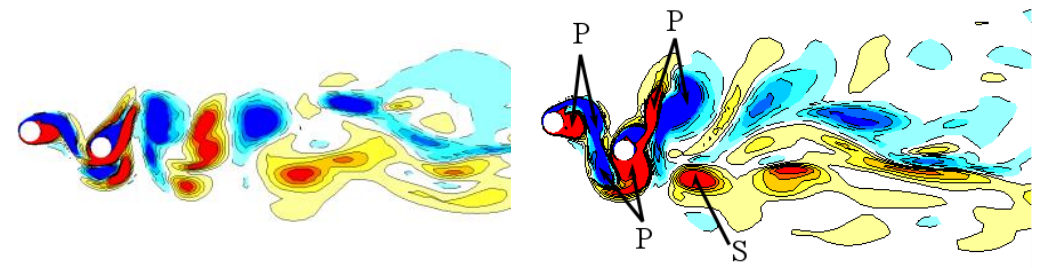

(b)

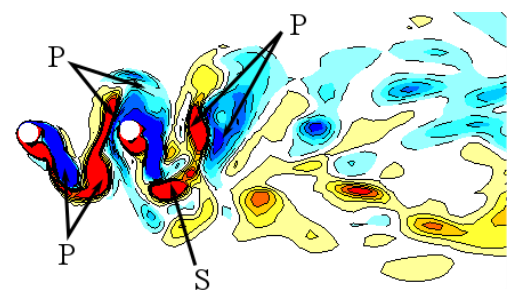

(c)
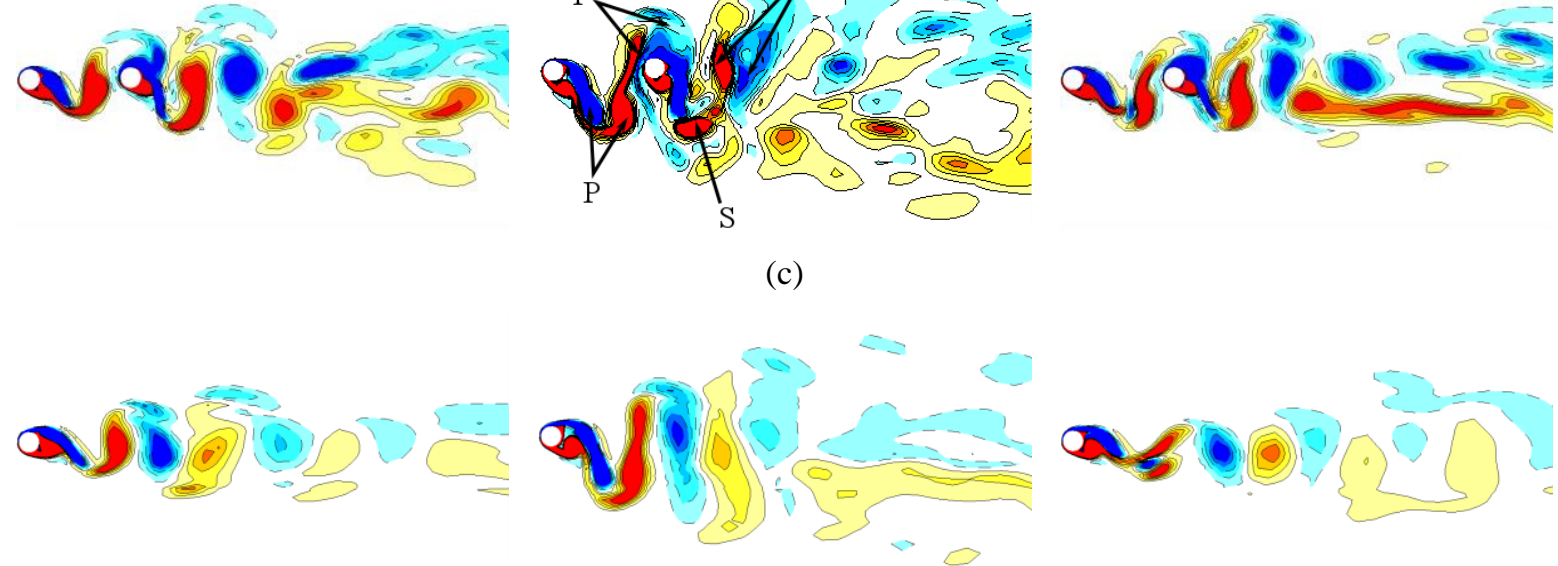

(d)

FIG. 21. Contours of spanwise vorticity $\omega_{z}$ on three different cross sections along the flexible cylinders at $V_{r}=6:$ (a) $S_{x} / D=$ 2.5 , (b) $S_{x} / D=3.5$, (c) $S_{x} / D=5$ and (d) single flexible cylinder. 
$t=0 T$
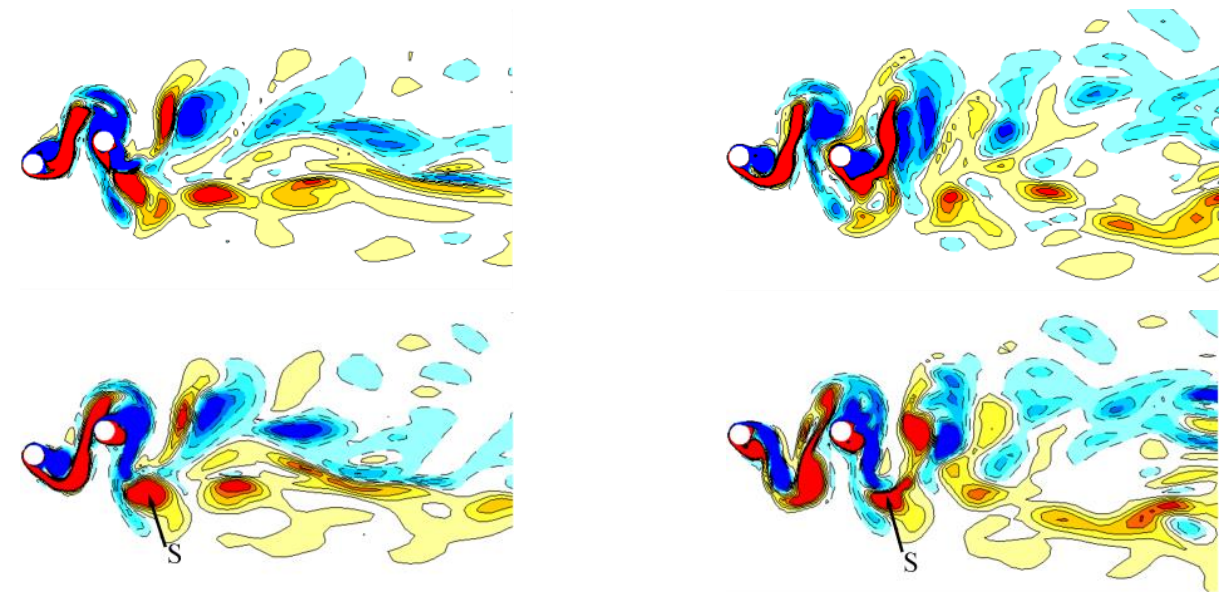

$t=0.25 T$
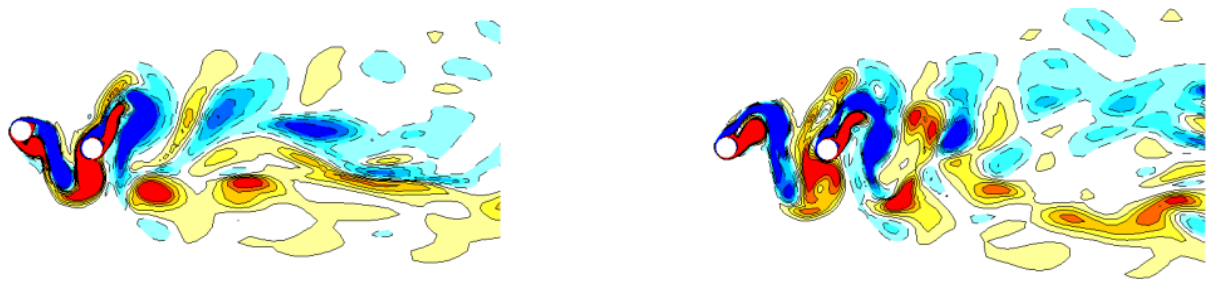

$t=0.5 T$
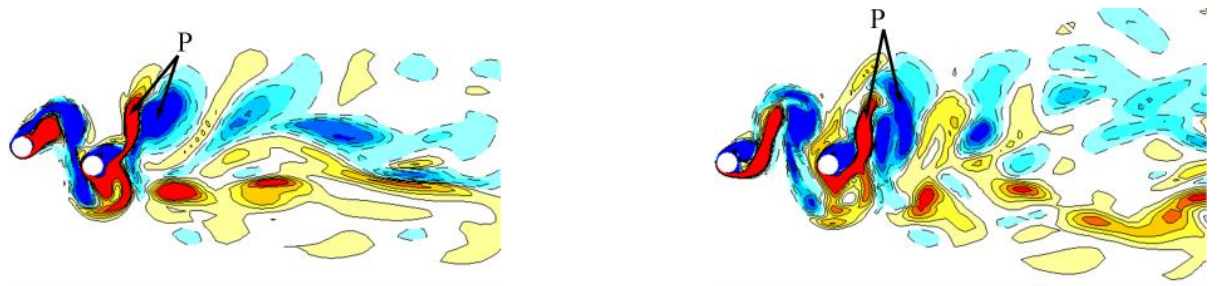

(a)

(b)

FIG. 22. Contours of spanwise vorticity $\omega_{z}$ on the middle sections of the two tandem flexible cylinders at different time instants in one vibration cycle when $V_{r}=6$ : (a) $S_{x} / D=3.5$ and (b) $S_{x} / D=5$. 


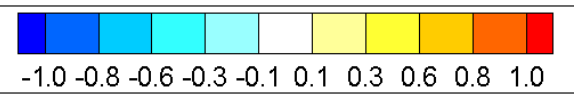

$z / L=0.25$

$z / L=0.5$

$z / L=0.75$
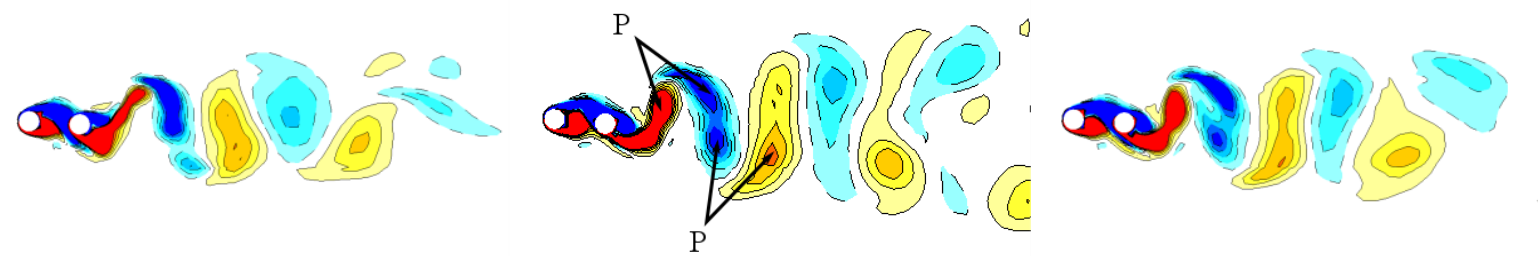

(a)
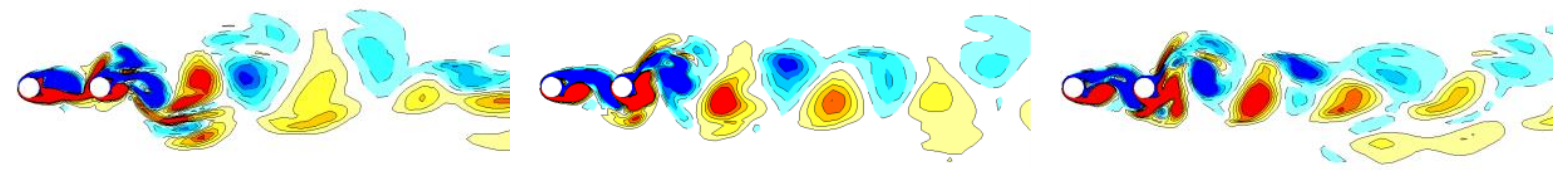

(b)
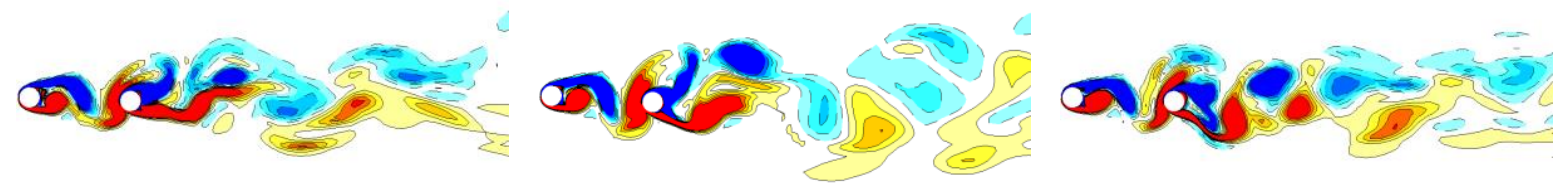

(c)
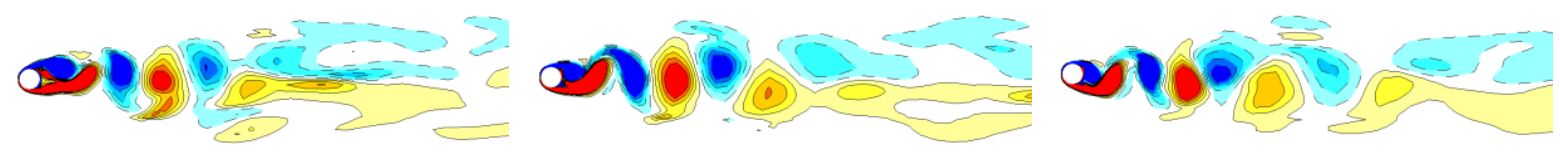

(d)

FIG. 23. Contours of spanwise vorticity $\omega_{z}$ on three different cross sections along the flexible cylinders at $V_{r}=10:$ (a) $S_{x} / D=$ 2.5, (b) $S_{x} / D=3.5$, (c) $S_{x} / D=5$ and (d) single flexible cylinder. 